\title{
Gradhiva
}

GRADHIV

Revue d'anthropologie et d'histoire des arts

$24 \mid 2016$

ARTchives

\section{Sampler les bruits de la ville, archiver les traces des balles. La création musicale hip-hop contre les politiques de l'amnésie au Gabon}

Sampling city noises, archiving bullet impacts: Hip-hop against amnesia policies in Gabon

\section{Alice Aterianus-Owanga}

\section{CpenEdition}

\section{Journals}

Édition électronique

URL : http://journals.openedition.org/gradhiva/3277

DOI : 10.4000/gradhiva.3277

ISSN : $1760-849 X$

Éditeur

Musée du quai Branly Jacques Chirac

Édition imprimée

Date de publication : 7 décembre 2016

Pagination : 108-135

ISBN : 978-2-35744-094-4

ISSN : 0764-8928

\section{Référence électronique}

Alice Aterianus-Owanga, «Sampler les bruits de la ville, archiver les traces des balles. La création musicale hip-hop contre les politiques de l'amnésie au Gabon », Gradhiva [En ligne], 24 | 2016, mis en ligne le 07 décembre 2019, consulté le 19 avril 2019. URL : http://journals.openedition.org/ gradhiva/3277 ; DOI : 10.4000/gradhiva.3277 


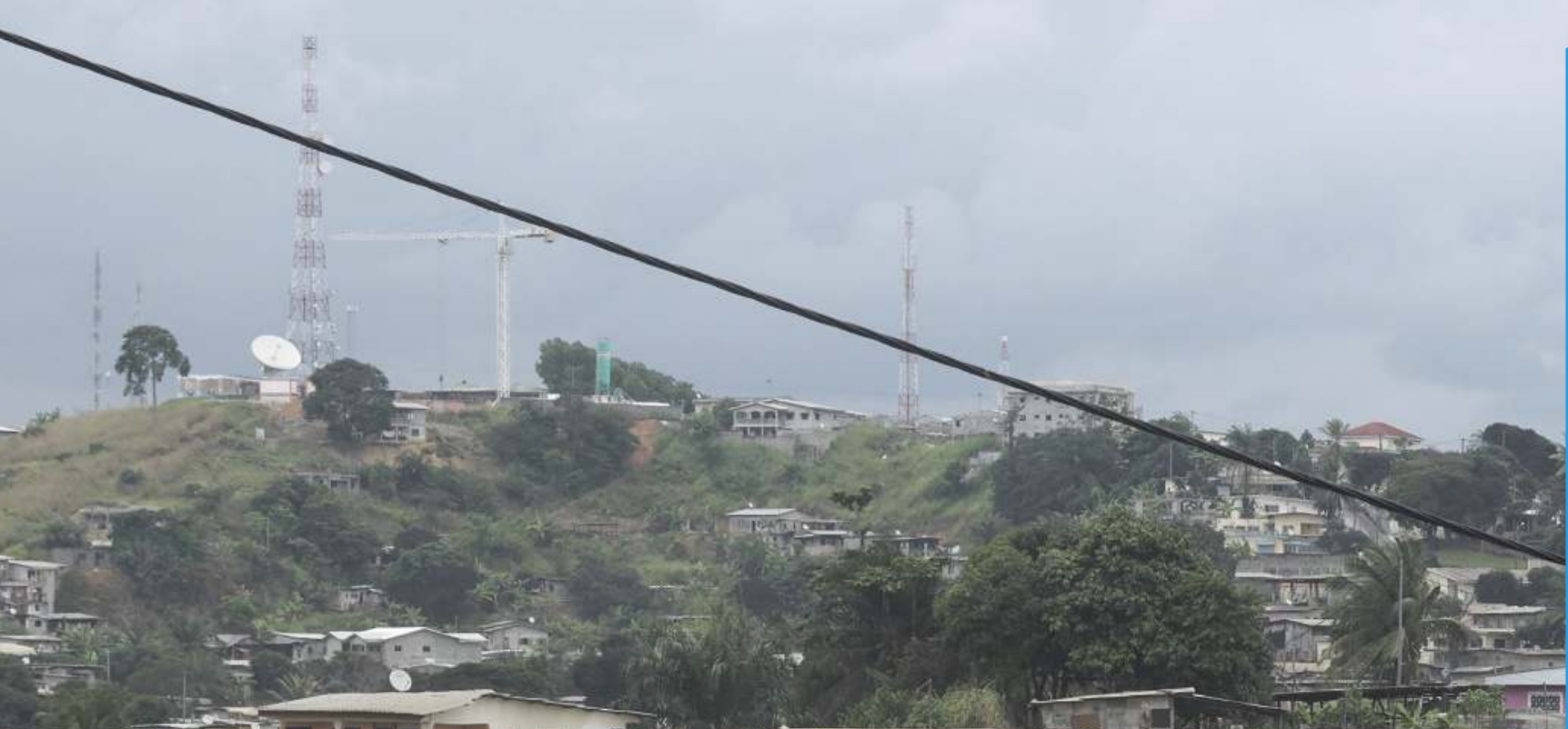

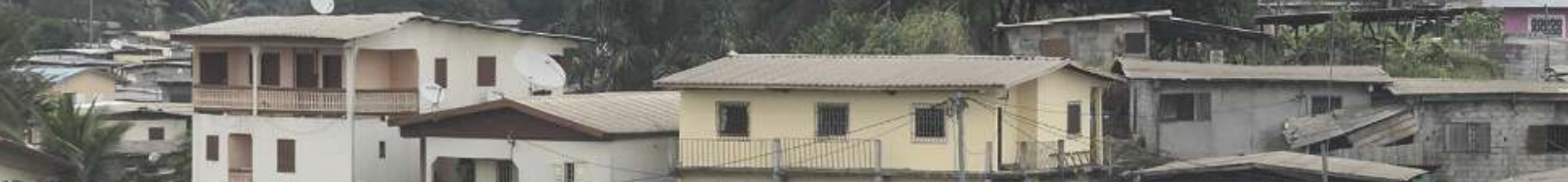

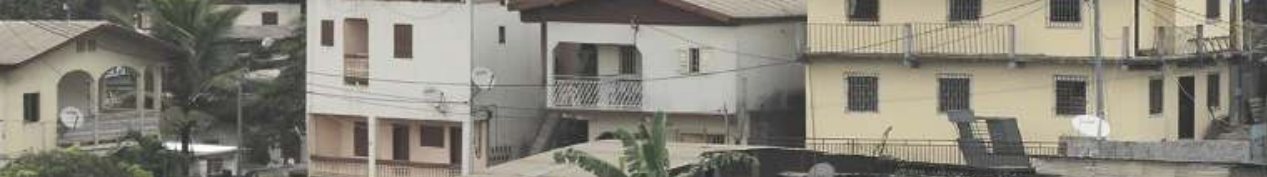

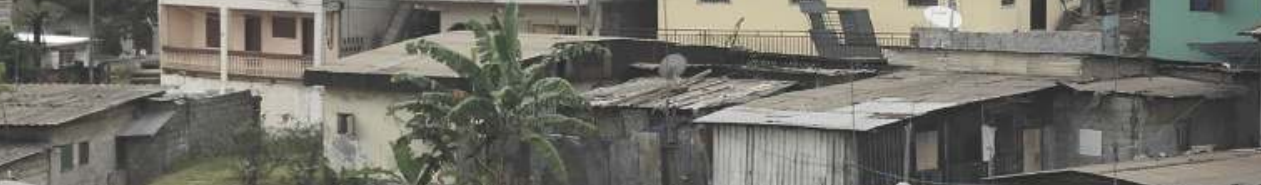

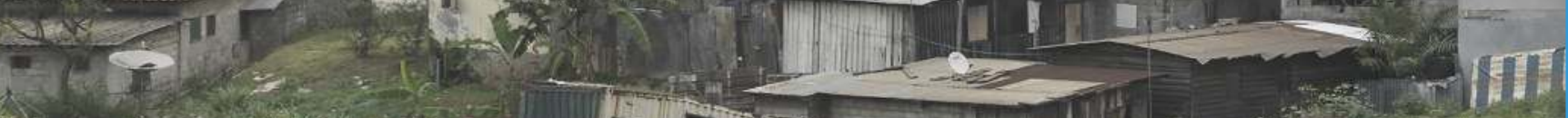

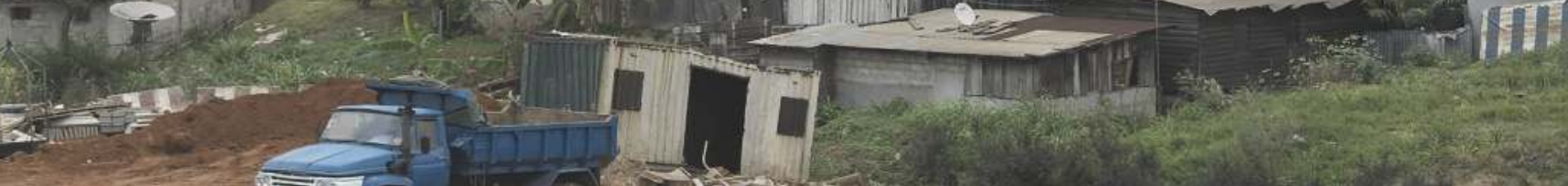

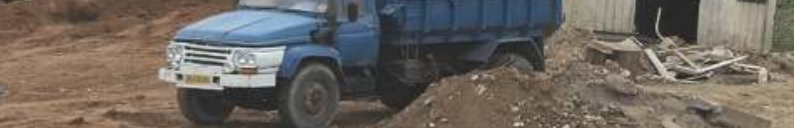

1)

-

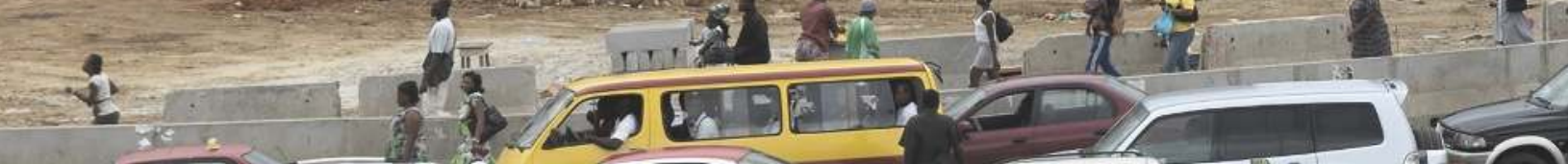

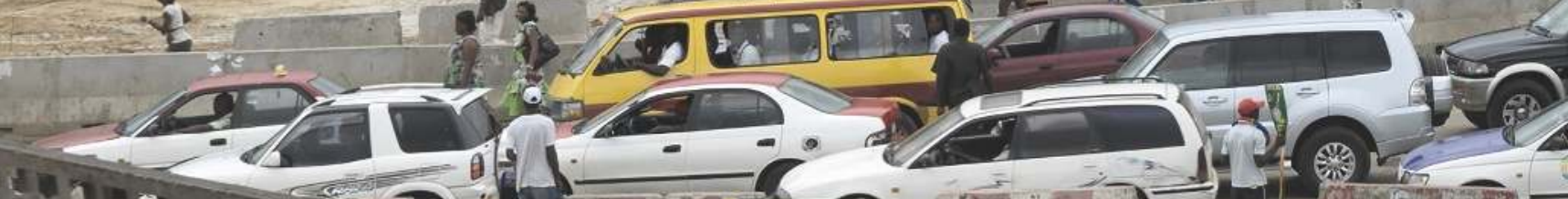

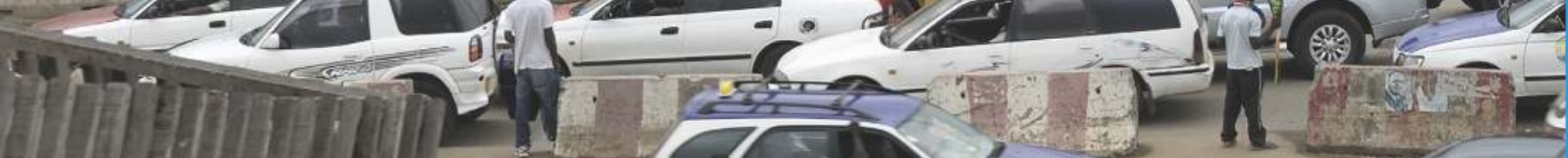

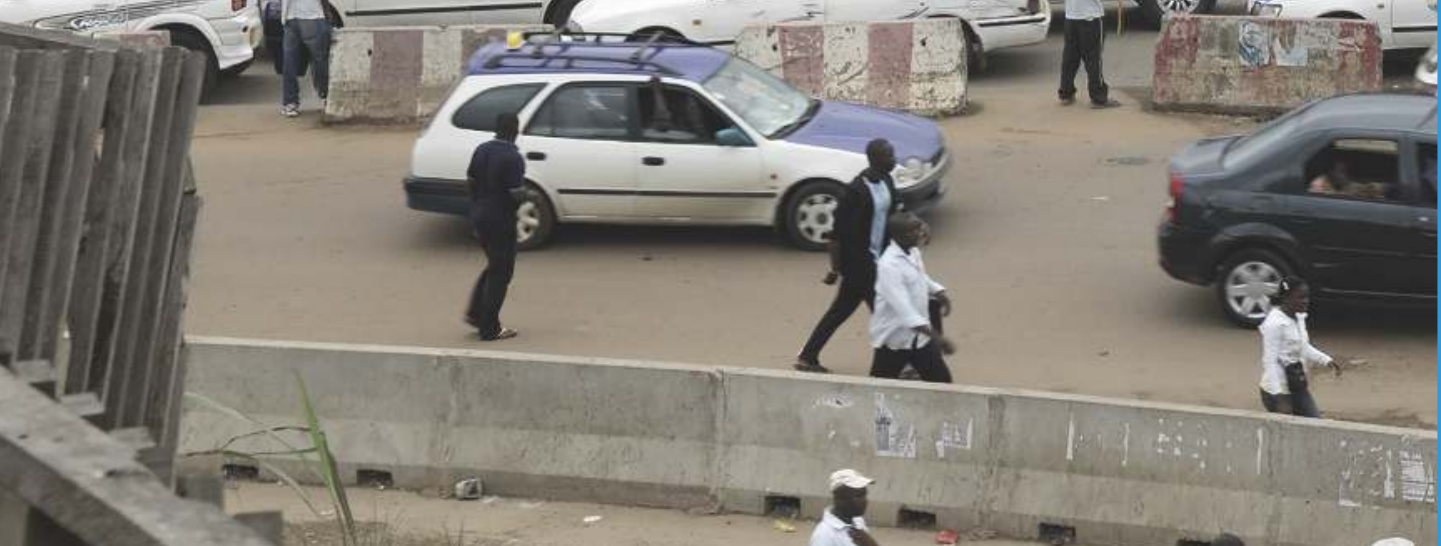

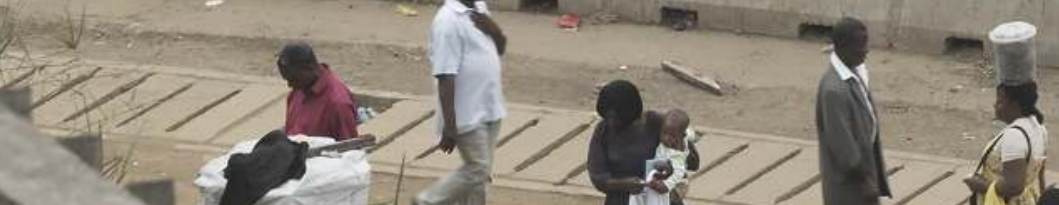




\section{Sampler les bruits de la ville, archiver les traces des balles}

La création musicale hip-hop contre les politiques de l'amnésie au Gabon

par Alice Aterianus-Owanga

Cet article examine les modes d'appropriation du sampling par les beatmakers du Gabon, dans un contexte d'occultation et de destruction des archives officielles. L'analyse des pratiques créatives, des discours idéologiques et du rapport à la notion d'archive chez plusieurs d'entre eux, fait apparaître que les usages de sonorités préexistantes répondent ici tantôt à des enjeux de particularisation, tantôt à un geste politique critique, dans l'intention de témoigner d'une expérience de la réalité sociale dissimulée par les politiques médiatiques. L'usage du sampling induit un geste créatif entrelaçant la resignification de sons du passé et l'archivage du présent, contribuant à la formation d'archives ordinaires. 


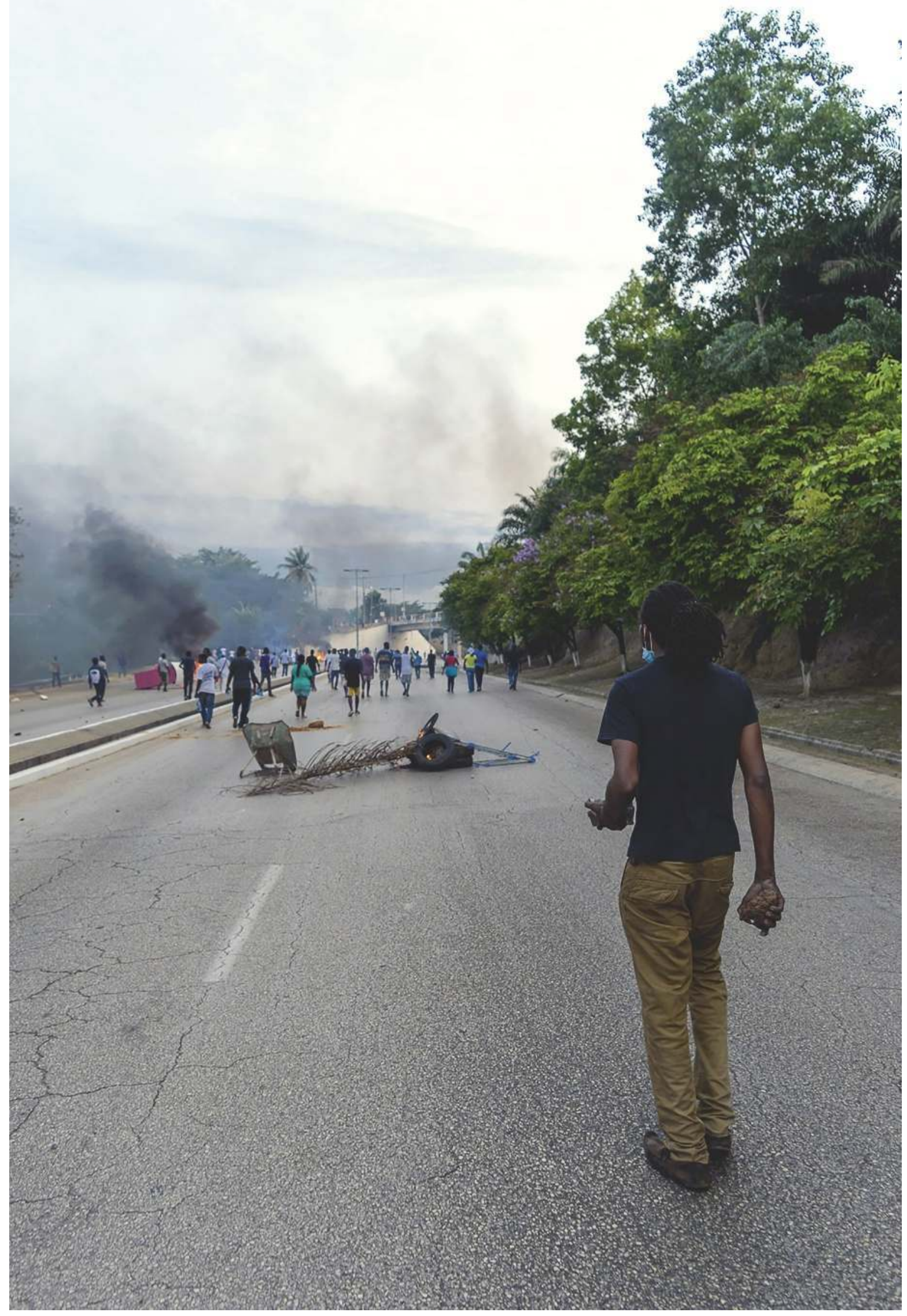


L'art du sampling - technologie de découpe et de transformation d'enregistrements sonores préexistants - est constitutif du hip-hop, et le chinage de matériaux sonores qu'il induit s'impose comme geste fondateur de nombre de créations musicales de ce mouvement. II en est non seulement l'un des soubassements technologiques, amené par l'usage du sampler, mais aussi un véritable choix esthétique, à l'origine d'une sonorité particulière (Béthune, 2003: 63-79, 2011; Déon, 2011; Harkins, 2012). C'est au travers d'incessants allers-retours entre des samples de musiques préexistantes et des enregistrements de sonorités ordinaires que les beatmakers créent la texture sonore caractéristique de l'esthétique hip-hop, reconnaissable par sa dimension cyclique. Le sampling participe en effet plus globalement d'un principe de composition par la mise en boucle (looping) d'une multitude de fragments sonores ${ }^{1}$, altérés et fondus dans de nouvelles harmonies:

«Quelle que soit la technique utilisée pour y parvenir, l'idéal du beatmaker est de créer une figure répétitive qui peut être altérée par l'addition ou la soustraction d'éléments variés en même temps. [...] Au niveau le plus basique, la mise en boucle (looping) réinvente automatiquement chaque matériau musical qu'elle mobilise, dans la mesure où la fin de la phrase est juxtaposée de façon répétitive avec son début, d'une manière qui n'était absolument pas voulue par le compositeur du morceau utilisé. [...] Si la mise en boucle ne change pas le son de la musique - son rythme, sa mélodie, son harmonie, ou son timbre -, elle change toute la sensibilité d'interprétation du son. Les mélodies deviennent des fragments musicaux (riffs). " (Schloss 2014: 136-138, traduction de l'auteure)

Au-delà du hip-hop, le sampling est une logique créative utilisée dans de nombreux genres musicaux. Les fragments sonores samplés sont parfois introduits pour ajouter une strate de signification symbolique au morceau (Martin 2010; Gaulier 2015), en se connectant à des genres musicaux précédents, mais sont aussi très souvent employés comme n'importe quelle autre matière sonore, indépendamment de liens revendiqués avec la source elle-même. Comme dans le cas de la musique techno (Kosmicki 2010), les compositeurs les transforment et les intègrent de façon méconnaissable dans de nouvelles œuvres musicales. Cette technologie multifacette véhicule ainsi des discours et idéologies très variables selon les genres musicaux qui l'exploitent, en fonction des contextes et des artistes qui s'en emparent (Harkins 2012).

Longtemps négligé au profit d'analyses du contenu discursif des textes de rap, le travail du sampling commence à être bien documenté dans les études sur les musiques hip-hop en Occident (Rose 1994; Béthune 2003; Déon 2011; Schloss 2014), qui s'y intéressent en partie pour ses enjeux esthétiques et les logiques créatives qui l'accompagnent, mais aussi pour ses dimensions mémorielles. Tricia Rose notait déjà dans son œuvre pionnière que le sampling permet de «revendiquer une histoire musicale noire et d'implanter les sons du passé dans le présent» (Rose 1994: 89). Plus récemment, Le Guern s'intéressait à la manière dont le sampling transforme les rapports entretenus avec le passé (Le Guern 2012), notant que «ce processus met
1. La brièveté des

fragments découpés est aussi liée à la nécessité de contourner les droits d'auteur des morceaux utilisés. À propos des rapports entre sampling et copyright, voir Marshall 2006 ; Déon 2011.

\section{ci-contre}

fig. 1

Orassio Crow, Le respect des pierres. Rond-point de la démocratie. Militant prêt à en découdre avec les forces d'intervention pour le respect de son vote. 21 août 2016 Libreville, Gabon. 
2. Il existe une ample littérature sur le rap africain. Pour quelques ouvrages collectifs récents sur la question, voir entre autres Charry (dir.) 2012; Saucier (dir.) 2011 ; Aterianus-Owanga et Moulard (dir.) 2016.

3. Le terme "beatmaker" désigne le concepteur musical dans la création hip-hop, celui qui crée les instrumentaux (beats) sur lesquels le rappeur déclame ses textes. Les beatmakers décrits dans cet article ont été choisis pour leur notoriété sur la scène musicale gabonaise et dans la diaspora, incarnant différentes tendances de la création musicale hip-hop gabonaise actuelle.

4. Mon étude se base sur une ethnographie de cinq années au sein du monde du hip-hop de Libreville (dans le cadre de ma thèse), précédées de plusieurs années d'immersion dans Libreville et ses groupes de hip-hop. Voir AterianusOwanga 2013. en jeu [...] non seulement notre façon de raconter des histoires, de produire de la narration sonore, mais plus fondamentalement notre sens de l'Histoire " (ibid. : 53). Pour cet auteur, la relation du sampling à la conscience historique se décline sur plusieurs niveaux: dans sa volonté de préservation des sons et des messages du passé, dans son adéquation avec le projet postmoderne de déconstruction des idéaux d'«auteur» et de linéarité du progrès esthétique dans l'histoire de l'art et, enfin, du côté des auditeurs, dans sa «combinaison de super-hybridité postmoderne à base de collages et d'espace-temps reconfiguré" (ibid. : 54). En reliant différents espaces et temporalités dans un même média, le sampler et le numérique seraient à l'origine d'un nouveau «registre post-historique et post-géographique » (ibid.), manifestant dans le contexte occidental les intrications entre la musique en régime numérique, la nostalgie et la «hantise de l'oubli » (Huyssen 2011, cité par Le Guern 2012: 54).

Si les relations entre nouvelles technologies de la composition musicale et enjeux mémoriels semblent désormais bien décrites dans le contexte culturel occidental, qu'en est-il dans d'autres aires culturelles ayant adopté le sampling? Quelles logiques créatives sont nées de la rencontre entre ces technologies et des artistes évoluant au sein de régimes mémoriels différents? L'usage du sampling dans la création hiphop y a-t-il aussi mis en branle les rapports à l'histoire et les supports de transmission de la mémoire? En d'autres termes, comment les jeunes concepteurs musicaux se sont-ils approprié le sampler en tant qu'outil de création musicale et technique d'«archivistique sonore sophistiquée » (Le Guern 2012: 53)?

Ma contribution propose de réfléchir à ces problématiques en partant des usages du sampling chez quelques acteurs du hip-hop du Gabon, entre Libreville et les territoires de la diaspora gabonaise. Comme dans d'autres pays africains ${ }^{2}$, la culture hip-hop rencontre un grand engouement dans cet État d'Afrique centrale, où elle est devenue un vecteur de mobilisation identitaire, politique et mémorielle. Le Gabon est également un cas intéressant du point de vue des articulations entre productions mémorielles et technologies de la création musicale. En effet, loin de cultiver un rapport nostalgique à sa mémoire et de conserver un corpus surabondant d'archives institutionnelles, le pays se caractérise plutôt par une pratique de l'occultation, voire de la destruction des archives officielles.

Cet article examine les modes d'appropriation du sampling qui se développent dans ce contexte et décrit les pratiques créatives de quelques beatmakers ${ }^{3}$ gabonais, mettant en exergue leurs discours idéologiques et leurs relations à la notion d'archive ${ }^{4}$. II ressort que leurs usages de sonorités préexistantes répondent tantôt à des enjeux de particularisation, tantôt à des intentions de critique politique, dans l'idée de "faire trace» d'une expérience de la réalité sociale dissimulée par les politiques médiatiques. L'usage du sampling entraîne un geste créatif entrelaçant la resignification de sons du passé et l'archivage du présent, qui contribue à la formation d'archives ordinaires. 


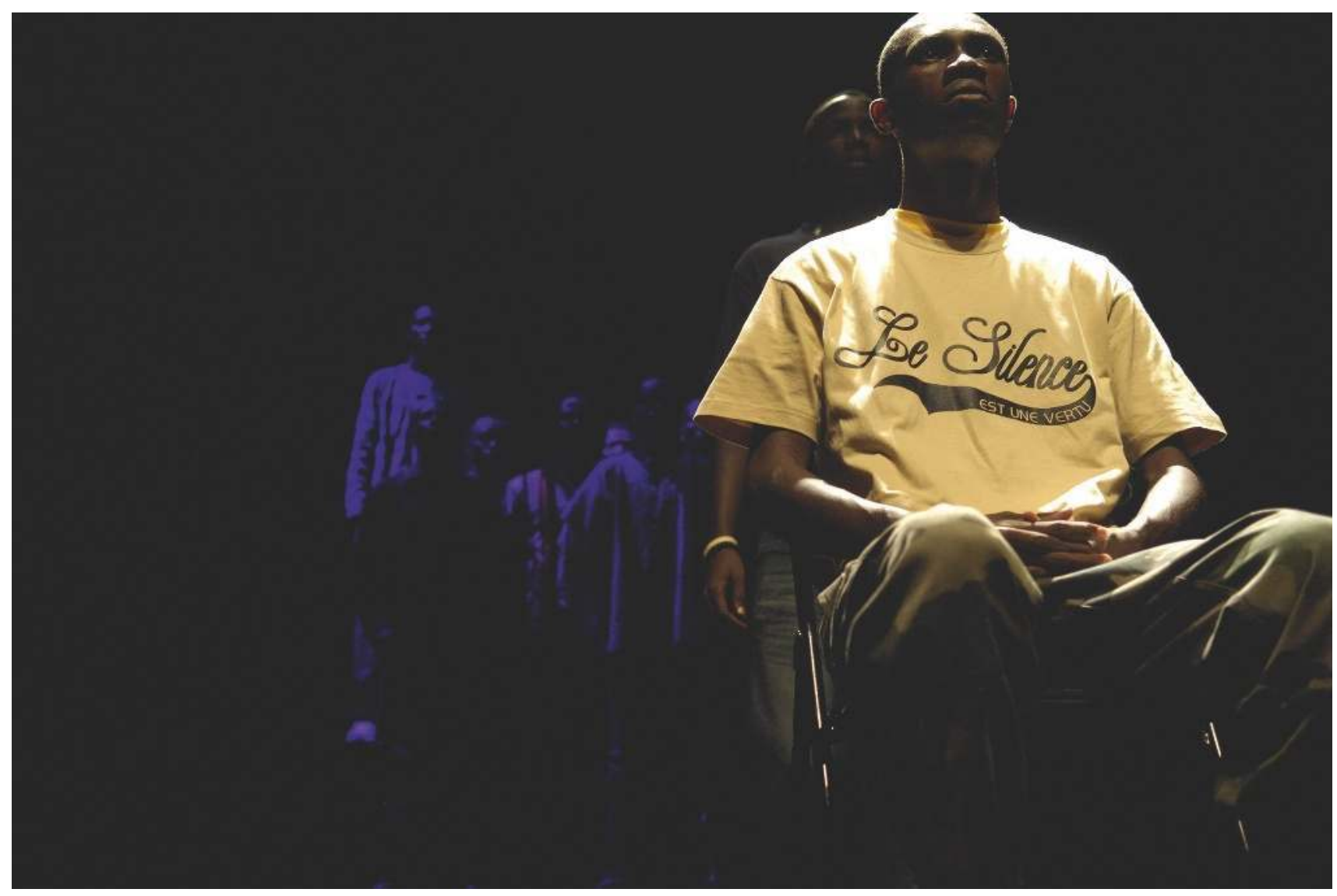

fig. 2

Larry Essouma, s.d.

(c) Xavier Bourgois. 


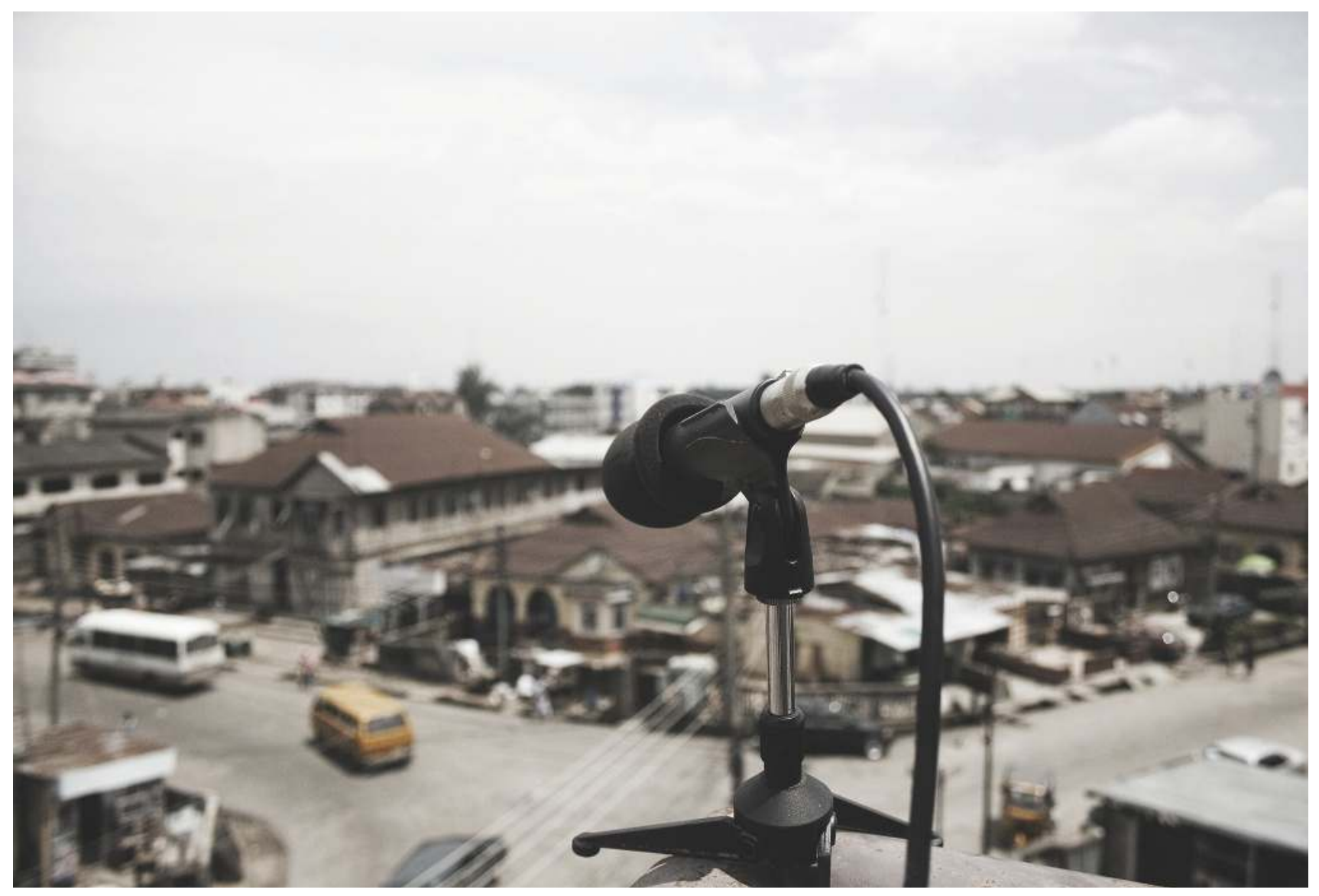

fig. 3

Prise de son à Lagos, s.d.

(c) Emeka Ogboh. 


\section{Des archives chronophages:}

\section{politiques de l'amnésie au Gabon}

Bien que le terme ait connu une migration sémantique depuis quelques années et se voie appliqué à tout un ensemble d'activités de conservation de documents, les «archives » sont avant tout l'œuvre et la propriété de l'État. Elles participent pleinement de sa construction en tant que "dépôt matériel de [sa] légitimité juridique et "constitutionnelle", et parfois historique" (Potin 2015: 7). Le geste d'archivage, de conservation et de requalification de documents comme archives comporte de fait d'importants enjeux de pouvoir, désormais bien connus (Foucault 1969; Derrida 1995; Stoler 2010). On sait notamment que par sa faculté à rappeler aux vivants la présence des morts et du passé, l'archive constitue une menace pour l'État, qui l'administre par la discipline ou par la destruction (Mbembe 2002).

C'est à ce second cas de figure que l'on serait tenté d'associer le Gabon contemporain lorsque l'on observe les lieux, les institutions et les acteurs en charge de la conservation des archives. En effet, les Archives nationales (créées en 1969) et sujettes à un état d'abandon et de détérioration (Messi Me Nang 2005), y sont fermées au public et aux chercheurs depuis plus de sept ans, en raison d'équipements défectueux et de l'absence de suivi dans le classement des fonds ${ }^{5}$. Par ailleurs, plusieurs opérations de destruction, d'effacement ou de disparition d'archives sont survenues de façon feutrée ces dernières années dans d'autres institutions. En 2009 par exemple, alors que la chaîne de télévision nationale déménageait et quittait ses anciens locaux, une partie de ses archives fut abandonnée dans le bâtiment désaffecté et mise sous surveillance militaire, devenant inaccessible au public. Plusieurs employés de la chaîne confient avoir assisté à la destruction partielle de ces fonds.

Cette pratique d'effacement des traces du passé se manifeste également par le sort que le gouvernement élu en 2009 a réservé aux monuments érigés depuis les indépendances. La Cité de la démocratie, créée à Libreville en 1977 pour accueillir le sommet de l'Organisation de l'unité africaine, et convertie par la suite en principale salle de congrès et de spectacles du pays, a ainsi été rasée dans le cadre de grandes opérations de reconstruction, au profit de l'édification d'un golf et d'un nouveau palais présidentiel.

Tout ceci rend compte du rapport des institutions gabonaises aux archives $^{6}$, illustrant les remarques d'Achille Mbembe sur ces États qui, face au pouvoir des archives, ont «tenté de les réduire au silence ou, de façon encore plus radicale, les ont détruites» (Mbembe 2002: 23, traduction de l'auteure). Dans le cas qui nous occupe, on peut y voir les symptômes d'une entreprise d'anéantissement des traces d'un passé récent, celui du régime d'Omar Bongo, président du Gabon de 1967 à 2009, auquel a succédé son fils Ali Bongo en août 2009.

Au-delà des archives officielles, le phénomène touche aussi les espaces de documentation de la capitale, comme l'université Omar-Bongo: la majorité des bibliothèques de départements y est désormais fermée à la consultation, et la bibliothèque universitaire centrale souffre d'un manque de renouvellement de ses fonds documentaires, parfois stockés à même le sol.
5. Ces informations sont issues d'une consultation personnelle des fonds documentaires de ces archives, grâce à des autorisations obtenues de façon exceptionnelle en août 2013.

6. Le quotidien national L'Union représente l'une des rares organisations aux ramifications étatiques qui conserve et rend accessible l'ensemble de sa documentation. 
7. À propos de la dette publique du Gabon, voir les statistiques mises en ligne sur le site de la Banque de France: https://www.banque france.fr/eurosysteme-etinternational/zone-franc/ series-statistiques-de-lazone-franc/zone-cemac. html. Voir aussi un aperçu dans cet article de la presse gabonaise en ligne: http ://www.gaboneco. com/retrospectiveeconomie-le-gabon-doitfaire-mieux-en-2016.html

8. La face $B$ des disques vinyles ou des cassettes de rap comportait auparavant les instrumentaux sans paroles des morceaux de rap ou les prestations a cappella, notamment pour que les DJ puissent utiliser les parties rythmiques dans leur mix. C'est aussi cette face $B$ des disques et des cassettes que les rappeurs amateurs utilisaient pour improviser sur les instrumentaux d'autres morceaux.

\section{ci-contre}

fig. 4

Freddy Tsimba, Aux arrêts, s.d. D.R.
Enfin, dans le milieu artistique et culturel, rares sont les artistes dont les archives personnelles (disques, photographies, presse, archives numériques) ont résisté à l'épreuve du temps, des inondations et des mobilités; quant aux traces matérielles des activités passées, elles ne font guère l'objet de conservation minutieuse, hormis chez quelques collectionneurs passionnés.

Sans qu'il soit possible de démontrer l'existence d'une action intentionnelle de suppression systématique des archives correspondant à un agenda politique explicite, ce désintérêt pour la question archivistique au Gabon peut, selon les historiens et intellectuels locaux, être interprété comme la résultante de deux facteurs: d'une part, il serait lié à une organisation culturelle privilégiant la transmission orale plutôt que la conservation écrite (Messi Me Nang 2005: 163); d'autre part, et de façon certainement plus vérifiable, il s'inscrit dans la continuité d'une absence de politique pérenne de soutien aux activités culturelles, intellectuelles et artistiques indépendantes de l'État (Engandja-Ngoulou 2012). Sur un plan contextuel, l'actuel délaissement des Archives nationales et des espaces de documentation correspond aussi à une situation de crise économique. Celle-ci se traduit par des dysfonctionnements, des grèves des institutions publiques et une augmentation considérable de la dette publique ${ }^{7}$, qui résulte, selon l'opposition, les intellectuels et les acteurs de la société civile, de la déprédation des ressources nationales par les autorités.

Une certaine désuétude de l'archive existe donc dans l'administration des archives du Gabon, témoignage d'un régime d'historicité "amnésique " et d'un État «chronophage» (Mbembe 2002: 23). Toutefois, bien que ces pratiques contre-mémorielles semblent rencontrer le consentement d'une large partie de la population, certains acteurs s'attachent également à les contourner par des pratiques ordinaires, notamment dans le monde du hiphop, où quelques artistes utilisent le sampler comme instrument d'archivage et de diffusion de leur vision de l'histoire.

\section{Du sampling dans le hip-hop gabonais: origines et étapes historiques}

Le hip-hop fait son apparition dans les boîtes de nuit et les résidences huppées de Libreville dans les années 1980, en grande partie via les voyages en France et aux États-Unis des classes aisées de la population. Ce sont avant tout les esthétiques et les attitudes corporelles des hip-hoppeurs français et américains que les jeunes Gabonais empruntent dans un premier temps, avant de s'essayer à imiter leurs expressions orales. Progressivement, et par le biais de solidarités de quartier des jeunes, les modèles de la culture hip-hop se transmettent depuis les classes aisées vers les classes populaires, déshéritées par la crise économique des années 1980 et par les répercussions du «système Bongo ». Portant les revendications qui accompagnent la transition démocratique des années 1980, le rap devient la caisse de résonance d'un désir de dénonciation des travers des post-colonies et, particulièrement pour le cas du Gabon, des inégalités violentes qui clivent la société.

Dans un premier temps, l'expression rap s'exerce sur les instrumentaux des "faces $\mathrm{B}^{\mathbf{8}}$ " des disques de rap français ou américains, ou sur des 

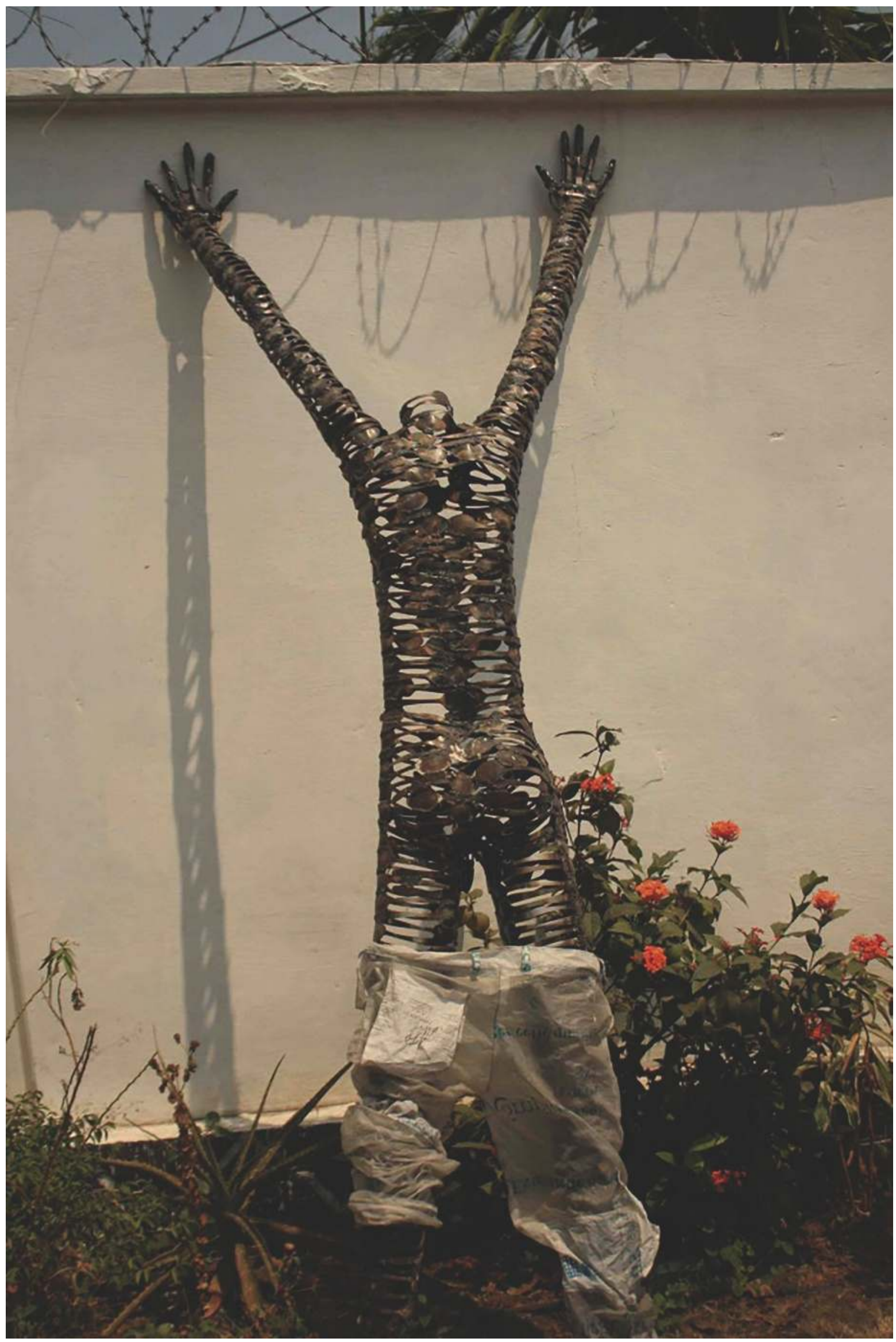


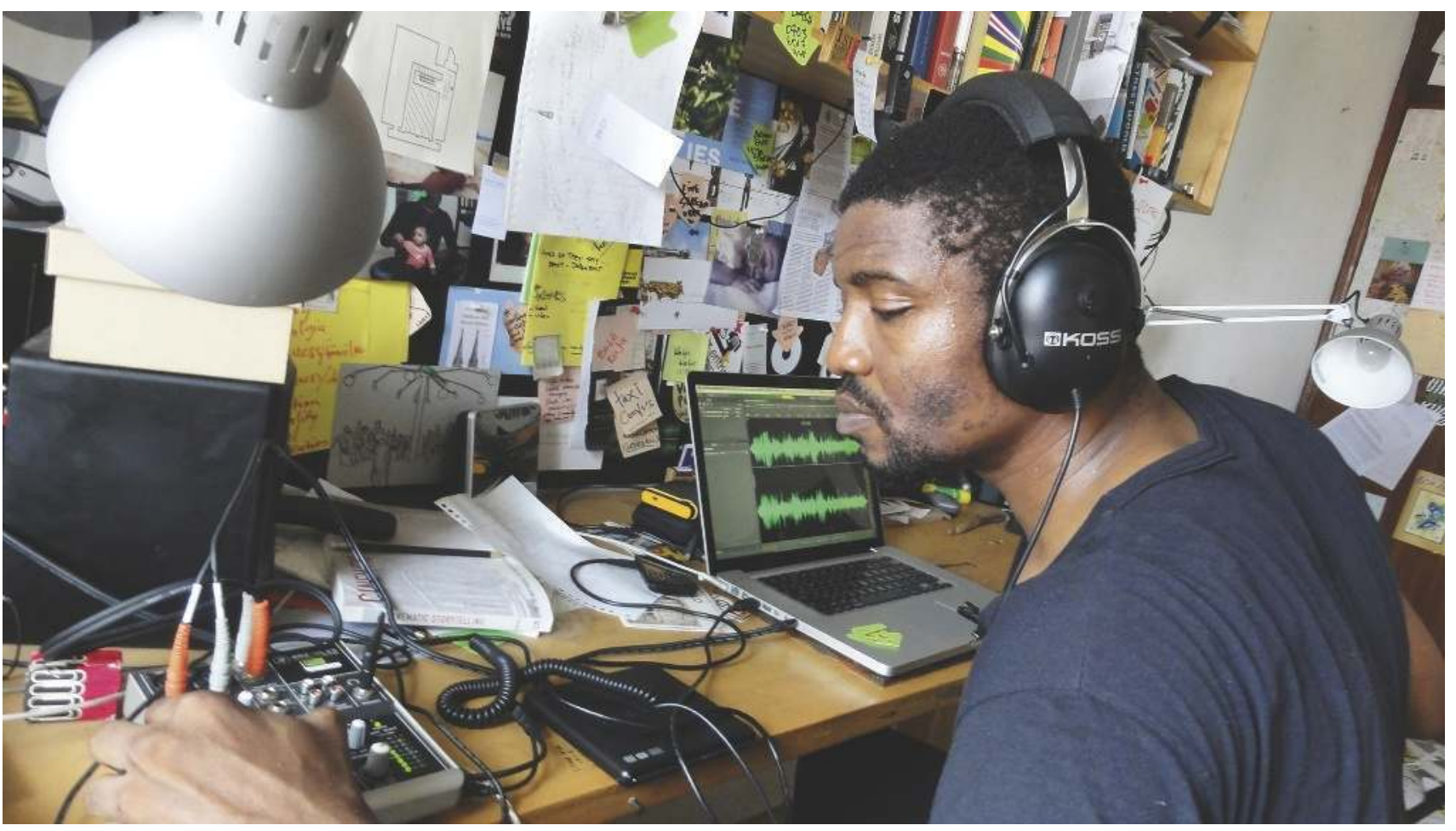

\section{fig. 5}

Emeka Ogboh, artiste sonore nigérian, 2013 (C) Hugues Jardel, Arte 2013.
9. Mapane Groove Act I, 1996, Jah Observer; Mapane Groove Act II, 1998, Jah Observer. boucles réalisées à partir d'extraits sans paroles de morceaux de rap. Seuls quelques groupes privilégiés ont alors accès à ces instrumentaux, et marquent une rupture par leur volonté de créer leurs propres beats qui particularisent le style du groupe et du rap gabonais. C'est le cas des deux groupes de rap localement précurseurs, V2A4 et Siya Po'ossi X. Le premier est un duo issu des classes aisées et des élites qui parvient à introduire des créations instrumentales originales du fait des voyages vers la France de ses membres. C'est là-bas qu'ils enregistrent leur premier album, dans des studios professionnels, ceux de Libreville n'accueillant pas encore les rappeurs de la capitale. La parution de titres de rap sur des beats originaux en 1990 va conférer au groupe V2A4 une primauté dans le champ de la création hip-hop et le consacrer, selon certains auditeurs, premier véritable groupe de rap gabonais.

À la même période, les membres du groupe Siya Po'ossi $X$, issus pour leur part de milieux sociaux très diversifiés, se dotent de leur première boîte à rythme (un séquenceur Sony) et s'essayent à la création d'instrumentaux électroniques. Progressivement, ils acquièrent davantage de matériel, et l'un des membres du groupe se forme au beatmaking auprès d'un ingénieur du son du Centre culturel français. Avec son double album, qui paraît en deux volets entre 1996 et $1998^{\circ}$, Siya Po'ossi X marque une seconde rupture dans le champ du beatmaking gabonais en introduisant pour la première fois des sonorités d'instruments traditionnels gabonais dans des beats de rap. Dans la lignée des musiciens tradi-modernes qui, depuis les années 1960, associent technologies instrumentales modernes et instruments traditionnels (Aterianus-Owanga, à paraître), les rappeurs et beatmakers de l'époque commencent à utiliser leur séquenceur pour sampler des mélodies de harpe ngombi, d'arc-en-bouche mongongo ou de mvett, 
qu'ils entremêlent de sons électroniques. Cette particularisation sonore est associée à une tentative de vernacularisation des registres langagiers, par un mélange des différentes langues des membres du groupe (Auzanneau 2001).

Comme Siya Po'ossi X, et parfois sous leur houlette, certains rappeurs s'essayent dès le milieu des années 1990 à élaborer des instrumentaux qui incorporent à la fois les sonorités mélodiques de leur environnement et des samples d'anciens titres de musiciens gabonais. L'artiste le plus samplé des beatmakers gabonais est ainsi le chanteur exilé Pierre-Claver Akendengué, connu pour avoir dénoncé le régime du parti unique (Tindy-Poati 2008; Retondah 2012; Aterianus-Owanga, à paraître). Un de ses textes a inspiré le refrain d'un titre célèbre de l'histoire du rap gabonais, "Aux choses du pays", qui décrit la précarité, l'insalubrité et les violences du quotidien des Gabonais ${ }^{\mathbf{1 0}}$. Le duo qui en est l'auteur, Movaizhaleine, est devenu depuis le milieu des années 1990 l'emblème de cette création nationale associant samples d'instruments gabonais, extraits du patrimoine musical local et autres fragments sonores: l'esthétique originale qui en découle répond aux désirs de création d'un rap à la fois caractéristique du Gabon et conforme aux canons de ce genre globalisé ${ }^{\mathbf{1 1}}$.

À l'heure actuelle, l'usage du sampling s'est raréfié chez les beatmakers de Libreville, dont on observe la préférence pour les rythmiques électroniques dansantes, réalisées à partir de boîtes à rythme, sans référence intertextuelle explicite mobilisant les «souvenirs sonores" des auditeurs (Bijsterveld et Van Dijck 2009). De fait, la majorité des beatmakers rencontrés et interviewés durant mon étude n'utilise que très peu les musiques préexistantes. Qu'est-ce qui motive cependant leur recours ponctuel au sampling? Ces artistes visent tout d'abord un objectif de particularisation par le sonore.

\section{Collages et sampling sonore: I'identification par le recours aux sonorités préexistantes}

Parmi les beatmakers contemporains, Owoninho ${ }^{12}$ est l'un des plus populaires auprès de la jeune génération de rappeurs et de chanteurs R\&B gabonais. Sa logique de création musicale offre un exemple intéressant: il explique que la banque de beats qu'il a constituée ne comporte qu'un faible nombre de morceaux basés sur des samples de musiques préexistantes (plus ou moins $10 \%$ d'après ses calculs) et que la plupart de ses créations relèvent davantage d'enregistrements qu'il puise dans une banque de données existante et met en boucle. Lorsqu'il incorpore des musiques préexistantes, la sélection des samples se fait en grande partie suivant une visée esthétique, en lien avec le rythme et l'ambiance musicale souhaités dans le morceau. Comme dans la musique techno (Kosmicki 2010), le beatmaker choisit le sample pour son timbre sonore, avant de le traiter et le transformer en un matériau parmi d'autres dans sa composition.

Toutefois, contrairement à la techno, la sélection du sample a ici une forte ambition «localiste». Owoninho emprunte volontairement de façon quasi exclusive aux morceaux d'artistes gabonais - tels que Pierre-Claver
10. "Aux choses du pays", Movaizhaleine, Mission akomplie, Zorbam Produxions, 2001

11. Cette contrainte de création d'un rap localisé se rattache à des débats d'authenticité qu'on retrouve sur de nombreuses scènes rap africaines (Charry [dir.] 2012), et dont le sens varie en fonction des contextes.

12. Issu d'une famille aisée et d'un père haut cadre dans l'administration politique, Owoninho a fait ses études au lycée français de Libreville, puis en France à partir de 2005, où il s'est lancé dans la production musicale en se formant de façon autodidacte. 
13. Le BPM ou battement par minute équivaut au rythme du morceau. Pour une description synthétique des techniques utilisées dans le sampling, voir Côté-Lapointe 2014.
Akendengué ou Oliver Ngoma - ou, exceptionnellement, à des titres de rumba congolaise, genre musical qui imprègne depuis les années 1950 les ambiances sonores de la capitale gabonaise et les souvenirs personnels d'Owoninho. Par ce travail de fusion de différentes sources, il ne s'agit pas seulement d'après lui de concevoir des structures rythmiques, mais de composer aussi de véritables harmonies musicales, en s'inspirant des musiques qui l'ont toujours accompagné.

Sur le plan technique, Owoninho crée ses samples par une opération de découpage d'un fragment n'excédant pas trois secondes, qu'il altère ensuite en les fusionnant avec d'autres éléments, par différents procédés: il inverse la séquence découpée, en supprime certaines fréquences (les basses le plus souvent), ou en accélère le BPM ${ }^{13}$. L'extrait initial est ainsi approprié, transformé et refondu dans la nouvelle composition, bien qu'il reste le plus souvent reconnaissable des auditeurs connaisseurs. Le titre "Eau bénite", basé sur un sample d'un célèbre morceau de rumba du chanteur Simaro, illustre ainsi l'effet décrit par Joseph Schloss dans son analyse du sampling (Schloss 2014: 138): les premières notes du sample déjouent les attentes de l'auditeur, puis la mise en boucle rompt avec l'œuvre d'origine et fait naître une nouvelle structure harmonique. Dans ce titre, la composition d'Owoninho débute par un extrait de la phrase de clavier inaugurale du titre de Simaro, à laquelle il ajoute rapidement un court sample de trompette capturé dans le même morceau, créant ainsi une première rupture par rapport au morceau initial. Puis, au bout de quatre mesures, il met en boucle cette phrase de clavier, y ajoute des claquements de main, des percussions et des battements. II finit par la redécouper en plus petits fragments, l'accélérer, la mixer, la répéter et l'inverser. Ce morceau sollicite alors la mémoire des auditeurs connaisseurs de rumba congolaise des années 1990 tout en s'affranchissant du titre utilisé, pour générer de nouveaux effets sonores et harmoniques.

Toujours dans l'idée de créer un son gabonais ou africain, mais cette fois sans recours à des musiques préexistantes, Owoninho compose aussi parfois des instrumentaux en enregistrant des claquements de main et des bruitages inspirés de rythmes traditionnels, qu'il met en boucle comme base sonore de ses beats. II en est ainsi de titres conçus à partir du rythme ikoku, pour lesquels il s'est imprégné de morceaux de groupes traditionnels du Sud du Gabon. Davantage que la liaison explicite avec les chanteurs qui l'ont précédé ou l'affirmation d'une idéologie nostalgique de retour au passé, c'est donc la recherche esthétique qui régit dans son cas le choix des samples, ainsi que la marque d'appartenance ou de localisme à laquelle renvoie l'extrait choisi.

Ici, comme l'ont déjà montré plusieurs travaux ethnomusicologiques (Guillebaud et Stoichiţă 2013; Déon 2011), la composition de nouvelles formes se développe à partir d'emprunts à des mélodies et des rythmes préexistants, qui sont autant d'inspirations, à l'inverse de toute approche dichotomique qui opposerait création et appropriation. Owoninho participe ainsi avec d'autres artistes de sa génération à la production d'une musique "afropop " à la gabonaise, qui entend affirmer une originalité musicale locale tout en suivant les canons populaires véhiculés par les musiques R\&B nigérianes (naija music), ghanéennes (hiplife) ou sud-africaines. Parallèlement, et sans 
que cela résulte d'une volonté explicite, ses compositions contribuent à réinjecter dans les paysages sonores urbains des extraits musicaux ancrés dans un passé proche, celui des musiques urbaines post-indépendances et de la génération antérieure de musiciens.

Au Gabon, en dehors de cette mouvance afro-pop, d'autres artistes inscrits dans la tendance du rap «conscient» emploient également les samples pour revendiquer une identité gabonaise, africaine ou noire, cette fois avec un objectif proclamé d'engagement critique, mettant en avant une vision alternative de l'histoire africaine. Ainsi, de nombreux beatmakers et rappeurs rencontrés font usage d'archives sonores dans le but d'affirmer une particularisation identitaire, en s'emparant des discours de figures du panafricanisme (Sékou Touré, Kwame Nkrumah, Thomas Sankara, Cheikh Anta Diop ${ }^{14}$ ), glanés sur la Toile ou dans des banques de données. Pour plusieurs artistes gabonais s'inscrivant dans la lignée des rappeurs américains afrocentristes, tels que X-Clan, il s'agit là de lutter par leur musique contre un impérialisme culturel et des lectures de l'histoire eurocentrées.

Hormis les références textuelles et les collages de citations, cette démarche de revendication d'un héritage africain ou panafricain passe également par un travail de composition musicale et par l'usage de fragments sonores ou musicaux non discursifs, intégrés, transformés et hybridés avec d'autres sonorités. La description du travail de composition d'un autre beatmaker et l'analyse d'une de ses créations musicales vont nous permettre d'examiner d'autres enjeux sous-jacents à ces créations, notamment dans le registre mémoriel.

\section{Le beatmaker en "alchimiste" du passé:}

\section{archivage et composition musicale chez Engone Endong}

Le DJ et beatmaker Engone Endong est l'un des rares compositeurs gabonais à accorder encore aujourd'hui une place prépondérante à la pratique du sampling. Pour lui, cette démarche répond en partie à une volonté pédagogique, celle de transmettre les messages et philosophies de générations musicales passées, principalement des musiques africaines et afroaméricaines dont il revendique l'héritage. Né en 1978 dans une famille aisée, formé en biologie à l'université des sciences et techniques de Masuku (USTM, dans l'Est du Gabon) avant de partir pour le Canada, il a appris le beatmaking en autodidacte. II s'est doté de matériel technique et d'une vaste collection de disques qu'il exploite pour composer ses morceaux ou animer des soirées, dans des clubs de Montréal ou des États-Unis, où il s'est fait connaître pour ses compositions hybrides, entre afrobeat et «world».

Ses collections de disques vinyles et de sons numériques sont ordonnées selon un classement méthodique: par continent, par pays et par titre d'album, puis par ordre alphabétique. Sur la grande majorité des disques vinyles, il inscrit aussi des annotations personnelles, qui décrivent les caractéristiques de chacune des pistes sonores du disque: $\uparrow$ pour tempo rapide, $\downarrow$ pour tempo lent, $\mathrm{PH}$ pour pitch ${ }^{\mathbf{1 5}}$ up, PB pour pitch bas, etc. Pour les besoins de la composition, il y puise des fragments sonores qui sont retravaillés dans ses samplers.
14. J'analyse plus amplement les constructions identitaires panafricaines ou afrocentrées des rappeurs gabonais dans d'autres travaux (AterianusOwanga, 2013, 2014).

15. Le terme "pitch» désigne la transformation de la vitesse musicale d'un morceau, technique particulièrement utilisée dans le beatmaking et le deejaying (également écrit "DJing ", pour évoquer l'activité de performance des DJ, ou deejays).

\section{double page} suivante

fig. 6

Guy Tillim, Libreville, 2012, diptyque, encre à pigment sur papier coton (C) Guy Tillim. Avec l'aimable autorisation de Stevenson Cape Town et Johannesburg. 


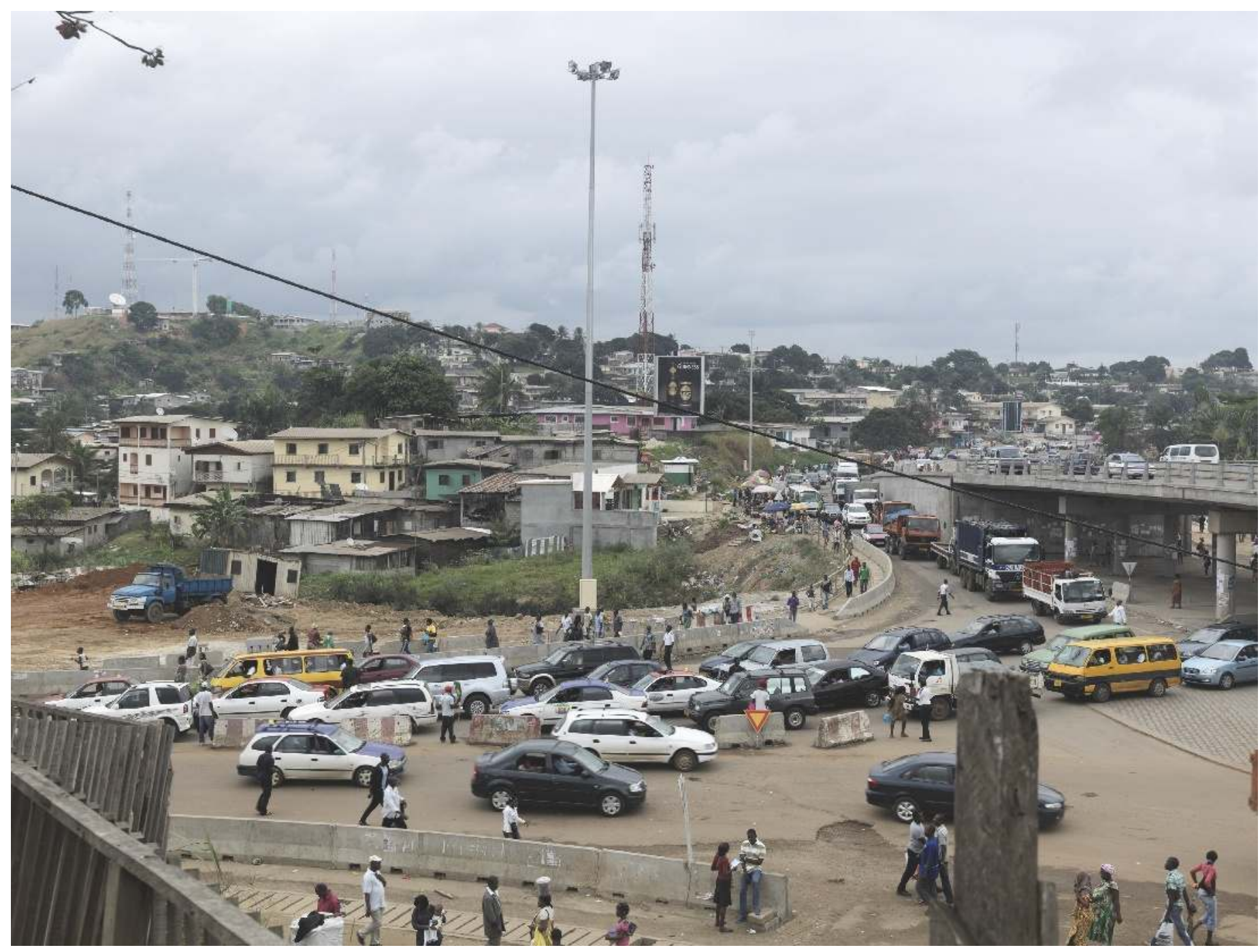




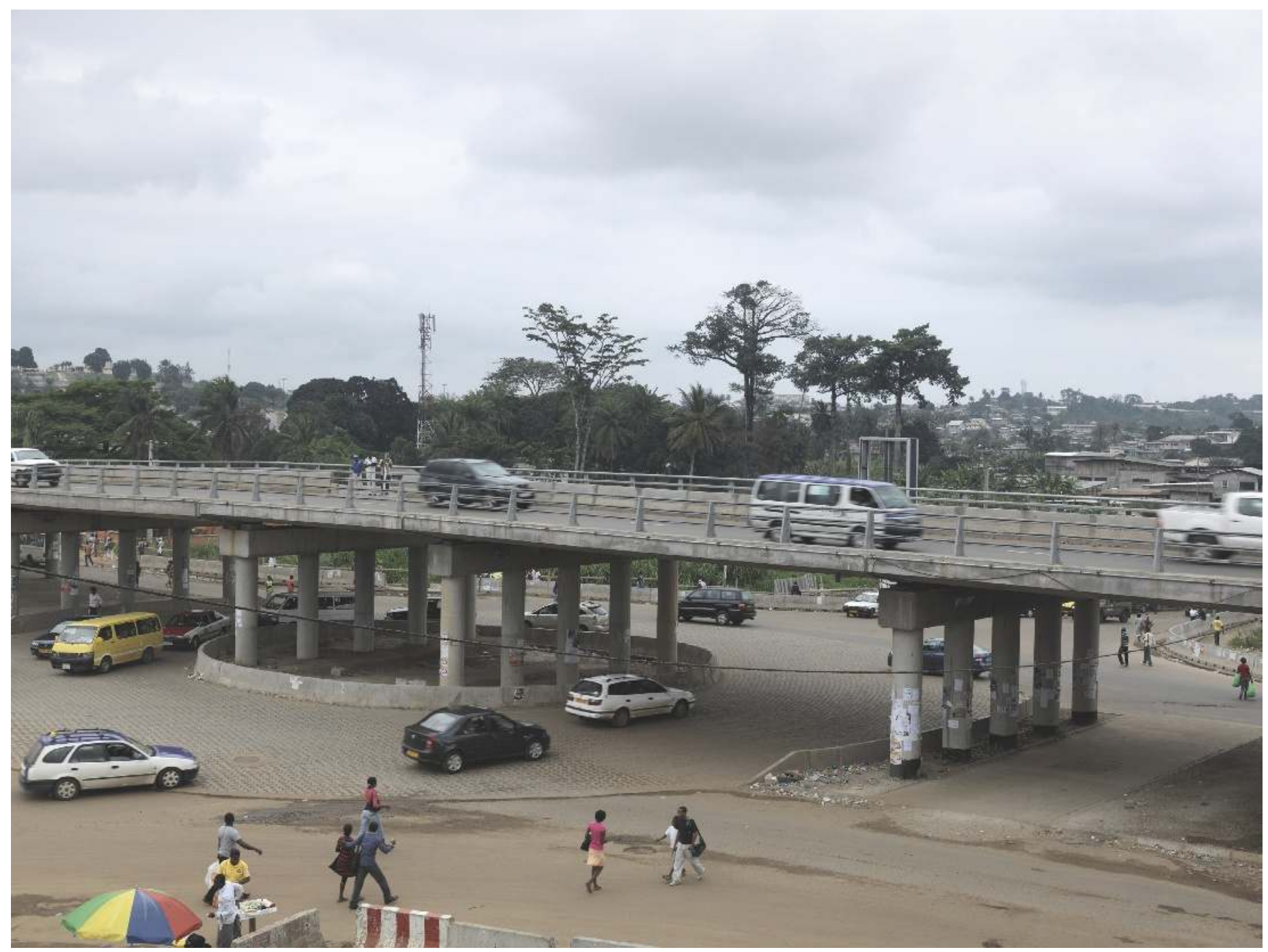


D'après Engone Endong, c'est grâce à sa collection et son érudition sur les musiques africaines que ses créations commencent à être appréciées et reconnues dans certains festivals de deejaying au Canada ou aux États-Unis. Sa démarche rencontre, dit-il, un écho favorable sur le marché nord-américain car elle répond à l'engouement actuel pour l'afrobeat et les sons africains. Portant un regard critique sur cette mode de l'Afrique en musique, Engone Endong tient pour sa part à accompagner son travail sur le son d'une véritable recherche sur l'œuvre de ces musiciens, leurs idéologies et les contextes de leurs créations musicales. Regrettant que l'attrait pour les musiques africaines ne rime pas toujours avec une révision des représentations stéréotypées de l'Afrique, il définit sa pratique musicale et son rapport au passé dans le beatmaking de la manière suivante:

Le travail de beatmaker, c'est de remanier tous ces trucs, de leur redonner vie, de remixer pour pouvoir donner un second souffle au truc. C'est des philosophies de vie. On ne peut pas avancer si on ne prend pas référence sur le passé. [...] Tu as des gens comme Fela, Tony Allen, c'est des gens qui étaient là et qui allaient chercher dans nos cultures et nos traditions. Pas parce que ça sonnait bien, mais aussi parce qu'il y a cette expérience de vie cachée à l'intérieur, c'est des leçons de vie aussi.

Donc moi, le rapport que j'ai avec le passé, c'est aussi pour le ramener. Pour qu'on puisse prendre appui sur ça pour pouvoir toujours être nous-mêmes. C'est comme un enfant, il a besoin de ses parents pour avancer. Dans la musique, c'est la même chose. (Entretien avec Engone Endong, New York, avril 2015.)

Pour Engone Endong, au-delà de sa dimension esthétique, le recours aux sons de musiciens précédents s'enchâsse donc avec l'affirmation d'une certaine vision de l'histoire à transmettre via la musique. Engone Endong procède pour ce faire à un méticuleux travail d'indexation et d'archivage de musiques et, à l'instar des DJ décrits par Philippe Le Guern, il se métamorphose lui aussi «en véritable archiviste de la musique» (Le Guern 2012: 52). Certes, dans les entretiens effectués, l'artiste refuse le terme d'«archives» pour qualifier les fragments de sons qu'il utilise, l'archive évoquant pour lui un «objet poussiéreux». II considère cependant que «le beatmaker fait aussi un travail d'historien» et qu'il s'apparente à «un alchimiste» du passé: c'est à partir des documents sonores glanés, collectés et conservés qu'il réinvente et transmet une connaissance sur l'histoire, pour le présent et pour le futur.

C'est pourquoi, même si les documents employés par Engone Endong ne peuvent effectivement pas être considérés comme des archives, la logique créative qu'il déploie s'apparente néanmoins à une forme d'archivage. Par son travail minutieux de collecte et de conservation de documents, il participe de fait à leur préservation et à leur classement, constituant des archives au statut hybride. Celles-ci relèvent en partie du registre des archives personnelles et ordinaires (Artières et Kalifa 2002) qui, créées dans le cadre du travail (ici de composition), témoignent du «geste du métier» (Artières et Laé 2011 : 5). Les collections ainsi élaborées appartiennent finalement, comme les albums photos, les journaux intimes ou les bibliothèques personnelles, 
à ces archives ordinaires et «populaires » décrites par Arjun Appadurai (2003), compilées dans le flux de la vie quotidienne et dépositaires d'une possible mémoire populaire. La description plus précise d'une des compositions musicales d'Engone Endong permet d'examiner le type d'enjeux que cette création d'archives ordinaires et personnelles soulève dans le contexte du Gabon contemporain.

\section{Recréer le "vacarme des villes": \\ le beatmaking comme art “mineur" subversif}

Depuis le début des années 2000, plusieurs productions hip-hop ont condamné le déni de mémoire et la censure qui occulte certaines pages de l'histoire gabonaise récente ${ }^{\mathbf{1 6}}$. En dehors du rap, cette intention critique et contre-mémorielle des créations hip-hop transparaît aussi dans les musiques composées pour les textes de slam, comme celui du slameur Larry mis en musique par Engone Endong en 2015. On y observe un remarquable jeu créatif entre le contenu discursif et la texture sonore, instructif sur les dimensions créatives et mémorielles du beatmaking.
16. Alors que le rappeur Johnny B-Good ravivait le souvenir des morts irrésolues d'opposants dans un titre nommé très à propos «Amnésie», le groupe Movaizhaleine faisait cas, dans le titre "Cour martiale", des violences politiques survenues à Port-Gentil suite aux mouvements de contestation de l'élection présidentielle. Voir le clip vidéo, disponible sur: https://www.youtube.com/ watch $? \mathrm{v}=\mathrm{qOC6UETQNNM}$.

\section{Extrait du texte de slam "Le vacarme des villes"}

Entendez le vacarme des villes. Entendez le tumulte de l'école.

Avec son infrastructure surannée, ses fonctionnaires mal payés,

ses grèves à répétition, sa corruption, ses résultats minables, et puis l'hôpital alité.

L'hôpital gorgé de palu qui n'en peut plus de lutter.

Entendez le vacarme des villes, cette misère qui ne désarme pas, avec sa dette, son pain sec, son riz blanc,

avec nos frères que l'alcool a délabrés, nos sœurs que la faim a déflorées, avec ses notables étriqués,

avec ses bars toute gueule ouverte qui distillent à s'en rompre les tympans la musique d'ambiance, et ces corps qui se trémoussent, ces corps qui se frottent et ces corps qui transpirent et ces corps qui puent, et ces vies qu'on ruine, entendez le vacarme des villes.

Entendez le crépitement affolé des gouttes sur la tôle qui hurlent les angoisses des aînés, et l'insouciance des enfants qui jouent nus sous l'averse.

L'eau lave, l'eau lave, mais c'est Dieu qui purifie.

Alors tous à la messe! Entendez le vacarme des églises, avec nos saintes, nos perfides.

Ils jactent, ils jactent,

ils espèrent que l'odeur de l'argent suffira à masquer la puanteur de leurs actes.

Entendez le vacarme des églises, avec nos mères qui prient encore et qui prient toujours du fond de nos taudis.

«Donnez la dîme! Le salut est à ce prix, leur a-t-on dit. »

Alors elles prient. Mais à force de cris, Dieu n'est plus audible.

Entendez le vacarme des églises, entendez le vacarme des villes.

Le père qui rentre usé et l'enfant qui braille, et la mère épuisée, qui regarde sans entendre

le journaliste qui dit au 20 heures que tout va bien, qu'il n'y a pas lieu de s'inquiéter,

entendez le vacarme des villes.

Ce texte fut déclamé pour la première fois par le slameur Larry ${ }^{\mathbf{1 7}}$ à Libreville en février 2015, en pleine période de troubles politiques. En effet, en décembre 2014, des manifestations de l'opposition avaient conduit à la 
18. Voir notamment l'article de Loic Ntoutoume «Un mort autour du meeting non tenu de Rio ", paru à l'époque dans Gabon Review [en ligne], disponible sur: http :// gabonreview.com/blog/ mort-autour-du-meetingtenu-rio/

19. Cette rumeur populaire, qui déclare que l'actuel président serait d'origine nigériane, a pris une ampleur considérable après la publication d'un ouvrage du journaliste Pierre Péan à l'automne 2014 (Péan 2014). Elle a occupé une large partie des discussions durant l'élection présidentielle d'août 2016.

20. Zora Group of Maputo in Music from Mozambique vol. 3, Folkways Records, 1983.

21. Traditional African Ritual Music of Guyana, David Blair Stiffler, Folkways Records, 1982

22. Disponible en ligne sur: http://www. lagossoundscape.com/

\section{ci-contre}

fig. 7

Freddy Tsimba, Silhouette effacée, s.d. D.R. mort d'un étudiant dans des circonstances non élucidées ${ }^{18}$. La contestation qui grondait alors dénonçait l'illégitimité du président Ali Bongo, soupçonné d'avoir usé d'un faux acte de naissance pour accéder à la présidence ${ }^{\mathbf{1 9}}$. Plus généralement, les manifestants s'indignaient contre la déliquescence des conditions de vie de la population dans les quartiers pauvres de la ville. C'est précisément la situation des quartiers déshérités et l'expérience ordinaire de la violence, camouflées par les médias officiels, que le texte de Larry invite à écouter, et que tente également de communiquer la musique quil l'accompagne.

Pour mettre ce texte en musique, Engone Endong a combiné une variété d'éléments acoustiques répondant aux propos du rappeur et se fondant en un arrière-plan censé restituer le "paysage sonore" (Schafer 1979) des quartiers de Libreville. Engone Endong explique ainsi le travail effectué pour ce morceau:

Le track de Larry, c'est le deuxième que j'ai fait [pour l'album dans lequel ce titre s'insère], et je me suis dit $\mathrm{OK}$, au lieu de créer un genre de musique, je vais créer une trame, un univers. Et pour essayer d'agrémenter, je me suis servi de tout ce qu'il y a comme samples sonores, j'ai pris tous les sons que je pouvais capter ici dans la rue, les klaxons, avec un micro. Et puis je me suis servi d'une banque de sons qu'un monsieur a présentée ici, aux États-Unis, du Nigeria; je me suis servi de ça aussi. Mais le truc le plus important, c'est de pouvoir suivre les mots. II était important de pouvoir aller dans son univers à lui. Ce que j'aime avec son morceau, c'est qu'il a une très belle plume; il a une manière de décrire la réalité. (Entretien avec Engone Endong, New York, avril 2015.)

En s'attachant à suivre le contenu discursif du slam, l'objectif d'Engone Endong est de produire "une trame" sonore qui accompagne et recrée I'univers narratif de Larry sur la vie ordinaire à Libreville. Or, il est intéressant de noter que cette recréation des ambiances sonores librevilloises passe par l'usage de samples issus pour majorité de sources bien éloignées du Gabon: pour ce morceau, Engone Endong a essentiellement utilisé des extraits glanés dans des disques de sa collection, dont un échantillon du disque Zora Drums ${ }^{20}$ et des extraits de chants du disque Traditional African Ritual Music of Guyana ${ }^{21}$. II a aussi fait usage de la banque de données sonores "Lagos Soundscapes", créée en 2013 par Emeka Ogboh, qui vise à capturer le paysage sonore de Lagos ${ }^{22}$. Enfin, il a lui-même enregistré des sons de son environnement, à Montréal. Pour la composition, Engone Endong a procédé à la mise en boucle de plusieurs de ces extraits sonores, des percussions notamment, en ralentissant ou en étirant certaines voix. À l'inverse, d'autres extraits n'ont subi qu'une faible altération, à l'instar des chants religieux. Ces sons aux provenances multiples n'ont pas finalement d'ancrage marqué dans la vie librevilloise et constituent plutôt une trame indépendante de toute attache territoriale spécifique.

Comment ce vacarme des villes, somme toute assez générique, en vient-il alors à nous parler de l'univers du slameur et des réalités locales librevilloises qu'il décrit? II semble que ce soit la capture de ces sonorités, 


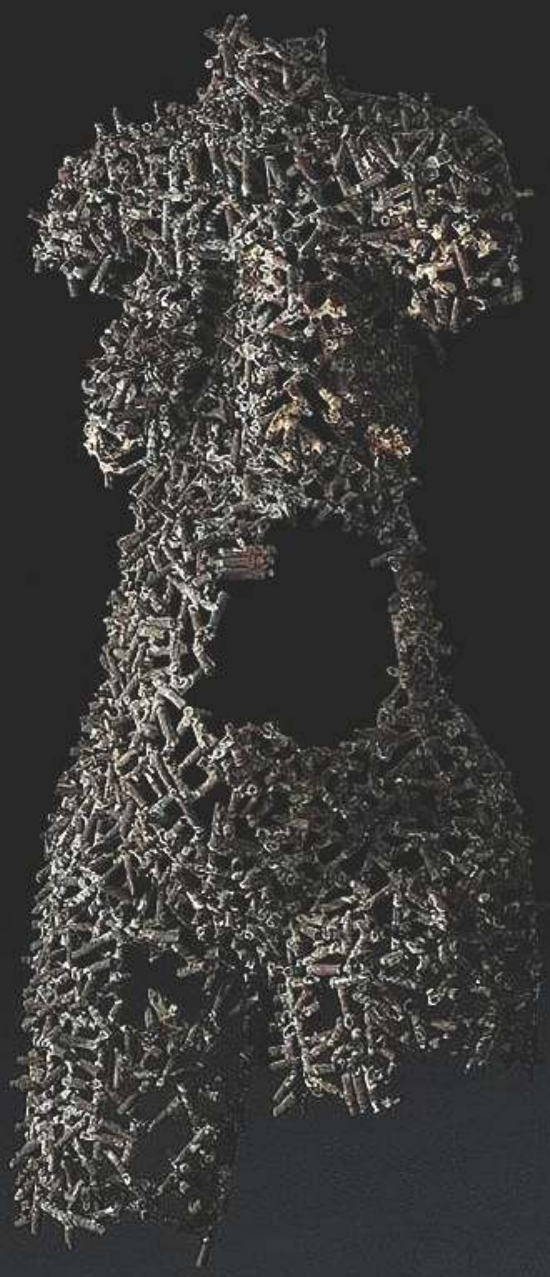




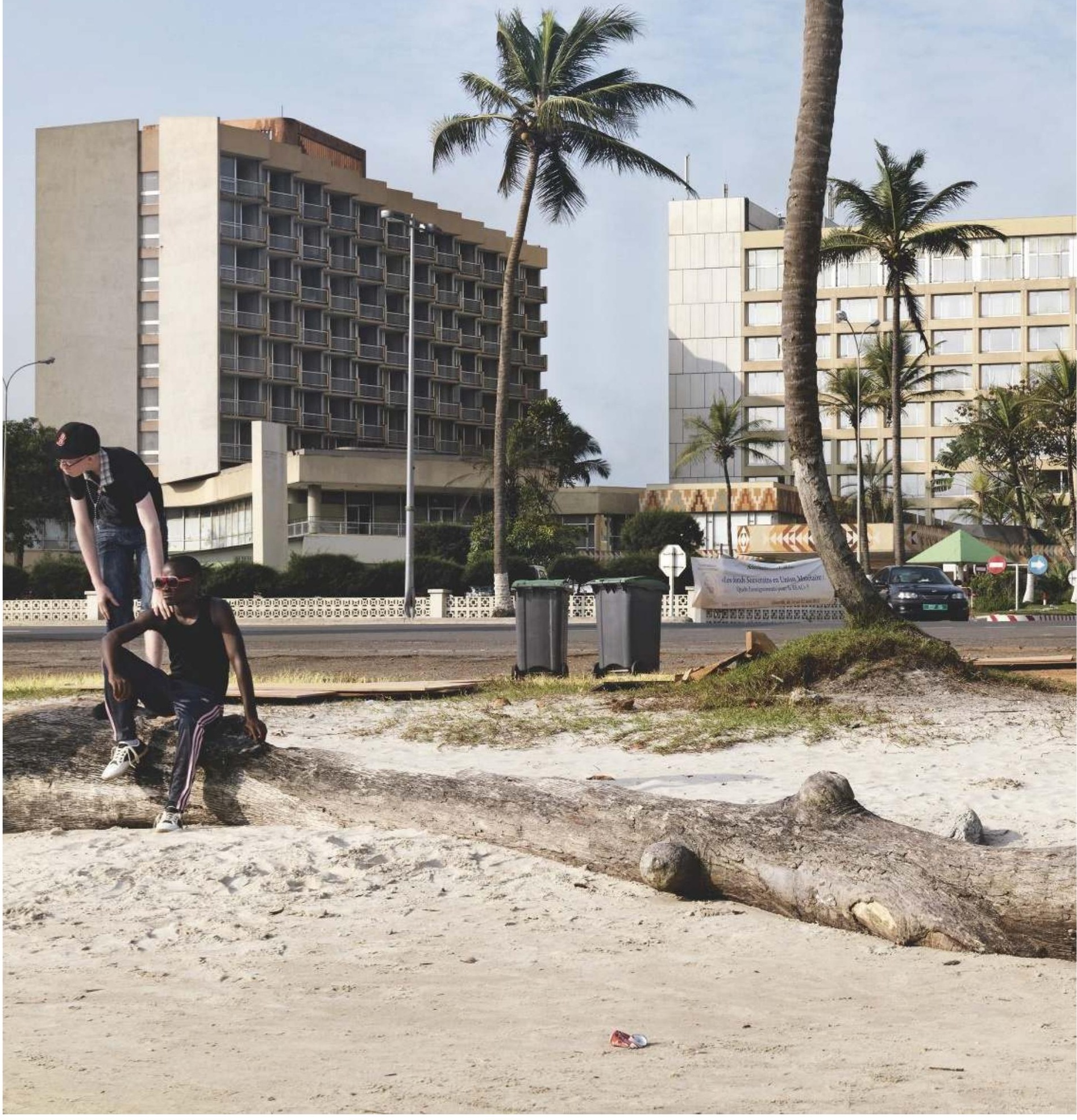




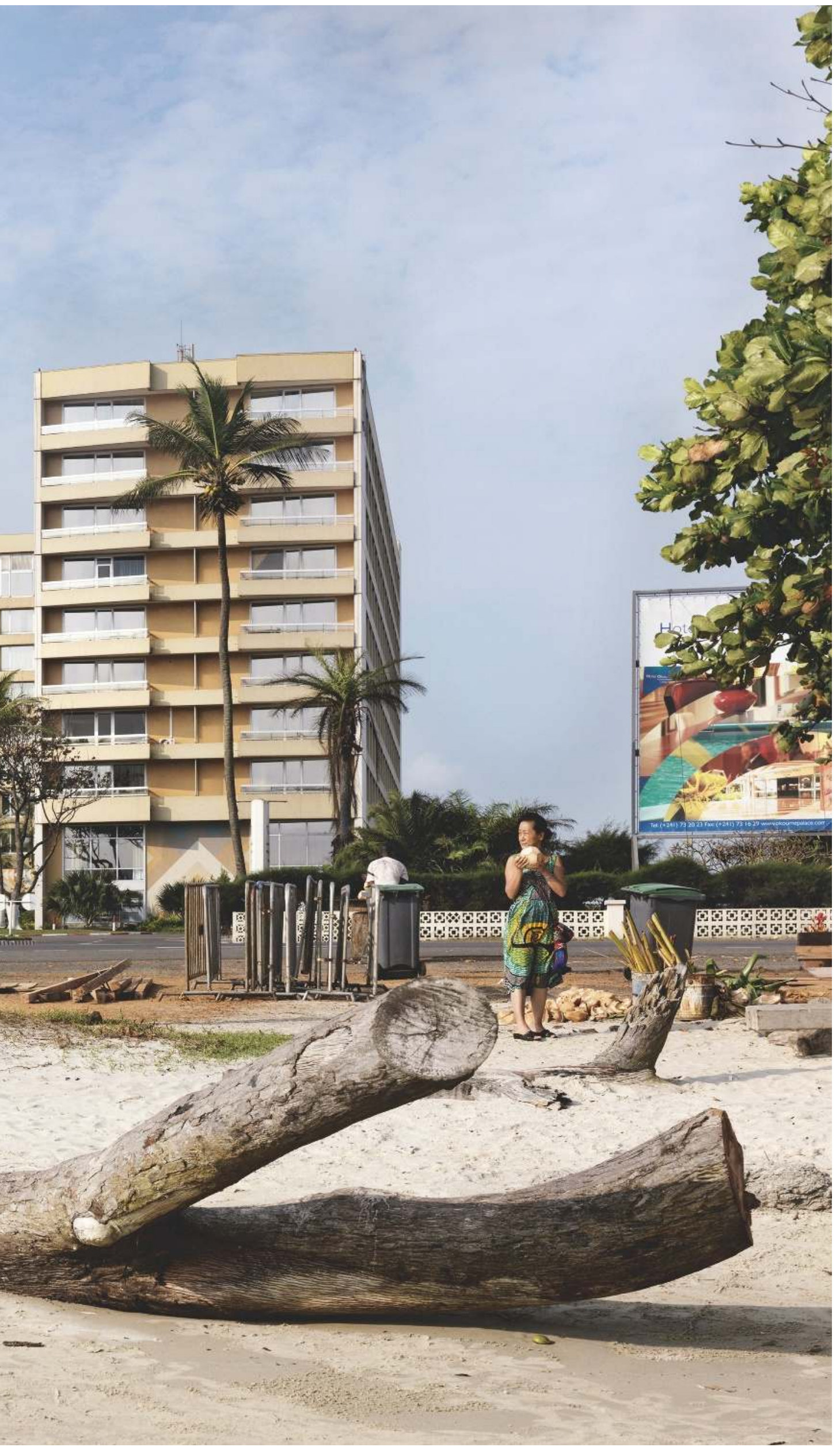

fig. 8

Guy Tillim. Intercontinental Hotel, 2012, encre à pigment sur papier coton (c) Guy Tillim. Avec l'aimable autorisation de Stevenson Cape Town et Johannesburg. 
23. Ce carrefour du sud de Libreville et le quartier qui l'entoure (IAl) doivent leur nom à l'Institut africain d'informatique qui s'y trouve, une école inter étatique de formation à l'informatique.

24. Plus récemment, des rappeurs ont fait paraître des titres pour menacer le président en place et le dissuader de briguer un second mandat. leur transformation et leur mise en relation avec la performance orale qui fassent de cette matière une possible incarnation du paysage sonore librevillois. En d'autres termes, la logique créative en présence disjoint les sources utilisées de leurs signification et origine initiales pour en faire des pâtes musicales au service de nouveaux sens. À l'écoute du texte de Larry, le crépitement des gouttes de pluie, les échos des chants religieux, le brouhaha urbain et les sonorités des klaxons deviennent de possibles empreintes de l'ambiance sonore de Libreville. Parfois méconnaissables, les sources sonores suivent ce processus de resémantisation par leur mise en dialogue avec les textes de Larry. En réalité, une majeure partie du texte dépeint des situations de précarité universelles, et ne comporte pas de références ou de toponymes le rattachant à Libreville. Comme ce texte, la composition sonore donne forme à un paysage musical urbain générique, dont la puissance réside à la fois dans le fait de s'ancrer dans la réalité sensible des espaces urbains populaires et dans la capacité à représenter une grande variété de villes africaines.

Si la plupart des samples ou des fragments sonores sélectionnés par Engone Endong ne sont pas identifiables, un dernier échantillon éveille cependant l'attention des auditeurs gabonais, choisi précisément parce qu'il leur est familier. Dans la partie finale du texte, où Larry mentionne le discours faussement optimiste du journaliste, Engone Endong a collé un passage d'un discours de Patrick Simangoye, animateur de télévision devenu célèbre pour ses tournures de phrase parfois alambiquées et sa conformation à la bienséance politique. Engone Endong a choisi un extrait télévisuel où le présentateur, parlant des détritus abandonnés dans l'espace public, déclare: «De quoi faire réfléchir les autorités municipales du $V^{e}$ arrondissement, avec cette ordure à poubelles placée en plein cœur du carrefour $\mid \mathrm{Al}^{23}$." Engone Endong a ici mis en boucle l'expression "ordure à poubelles", insistant ainsi sur les défauts de langage du journaliste pour mettre en exergue l'absurdité des politiques médiatiques du régime et ridiculiser les représentants de l'État sur un ton parodique.

Le morceau «Le Vacarme des villes» médiatise ainsi une expérience de la réalité sociale niée par les appareils idéologiques et médiatiques du régime en place. La composition sonore contribue à la formation d'une expression artistique «mineure», caractérisée par ce principe "d'immédiateté politique" qu'ont décrit Deleuze et Guattari (1975), ou plus tard Christian Béthune (2011). Dans ce titre, comme dans d'autres morceaux de hip-hop, «le bruit véhicule, en tant que tel, un immédiat politique» (ibid. : 176): par sa cadence et ses échos envahissants, le vacarme des villes s'avère "presque à lui seul investi d'une efficace subversive» (ibid.).

\section{Ironie et dérision: quelques modes mineurs de la critique par le sampling}

Au-delà de ces subversions «mineures», d'autres compositions hiphop ont fait usage du sampling pour dénoncer plus directement les dysfonctionnements du régime en place, la violence de la répression et l'exercice illégitime du pouvoir ${ }^{\mathbf{2 4}}$. Alors que des rappeurs rendaient hommage à la mémoire des morts passées sous silence durant les émeutes de Port-Gentil, 
ou à la disparition de l'étudiant Mboulou Béka durant les manifestations de décembre 2014, des beatmakers comme Lord Ekomy Ndong (membre du groupe Movaizhaleine cité plus haut) ont abordé la question du détournement de fonds publics par les élites au pouvoir, en diffusant sur le Net des créations sonores «vierges", sans performance verbale superposée. Dans le morceau «600 milliards ${ }^{25}$ », ce beatmaker confronte des extraits de discours d'Ali Bongo à ceux de ses opposants, procédant à un détournement parodique ${ }^{26}$ de fragments sonores mixés et mis en boucle sur un rythme rapide et une mélodie dansante. Jouant sur l'humour et la dérision qui font la marque du groupe Movaizhaleine, le titre comporte aussi des samples de la voix de Lord Ekomy Ndong et d'extraits de ses anciens morceaux qui critiquaient déjà les dépenses somptuaires des autorités. La dimension burlesque repose sur les altérations des extraits sonores choisis (mis en boucle) et sur la combinaison d'une rythmique dansante aux contenus politiques des discours collectés.

La parodie recourt aux nouveaux lieux de la critique politique qui se développent sur les réseaux sociaux: plutôt que des discours officiels d'opposants, les extraits samplés par Lord Ekomy Ndong proviennent des interventions de membres de la société civile et de la diaspora gabonaise postés sur Internet, lesdits Tata Huguette et Landry Amy Washington. Ces citoyens gabonais devenus célèbres utilisent YouTube et Facebook pour diffuser fréquemment des prises de position emportées, fustigeant la politique d'Ali Bongo et la famille présidentielle. De courts samples de certains de leurs discours, truffés d'expressions typiquement gabonaises (comme «ça c'est qui? » ou «on a vu le corrigé de ça ${ }^{27}$ »), sont incorporés au mix, diffusés via SoundCloud et YouTube, puis partagés sur Facebook et Twitter.

Le sampling permet ici une écriture à plusieurs voix, aboutissant à une création qui assemble divers documents enregistrés et collectés, pour garder une mémoire des faits et donner une visibilité aux discours contestataires qui s'élèvent au sein de la société civile.

\section{Le beatmaker en archiviste, le beat en archive}

Cet article s'est attaché à décrire la place du sampling dans le hiphop gabonais et la manière dont cet art éminemment «archival » (Foster 2004) a été adopté dans un pays où les archives (officielles et étatiques) font défaut. L'usage des technologies du sampling, chez les artistes évoqués ici, se fait au travers de rapports diversifiés au passé, parfois en dehors de toute revendication historique explicite et de tout engagement idéologique, d'autres fois dans une nette référence au passé comme acte de résistance à un régime politique chronophage et à une culture de l'amnésie. Comme le remarque Maxence Déon (2011), les beatmakers gabonais utilisent ici aussi le sampling en tant que véritable outil de création esthétique et de composition musicale. Parallèlement cependant, pour nombre d'entre eux, cette composition par appropriation sélective de sources sonores répond à des enjeux de particularisation de leur style musical, revendiquant leur appartenance au Gabon, à l'Afrique ou plus largement à la diaspora noire.
25. Voir le clip vidéo, disponible en ligne sur: https://www.youtube.com/ watch ?v=ftTkuAYd7WQ

26. À propos des différentes formes d'humour en musique, voir Loriot et Roubet 2011 (dir.); Guillebaud et Stoichiţă 2013.

27. L'expression «le corrigé " désigne dans le français du Gabon la version améliorée de quelque chose ou le niveau optimal d'une action. 


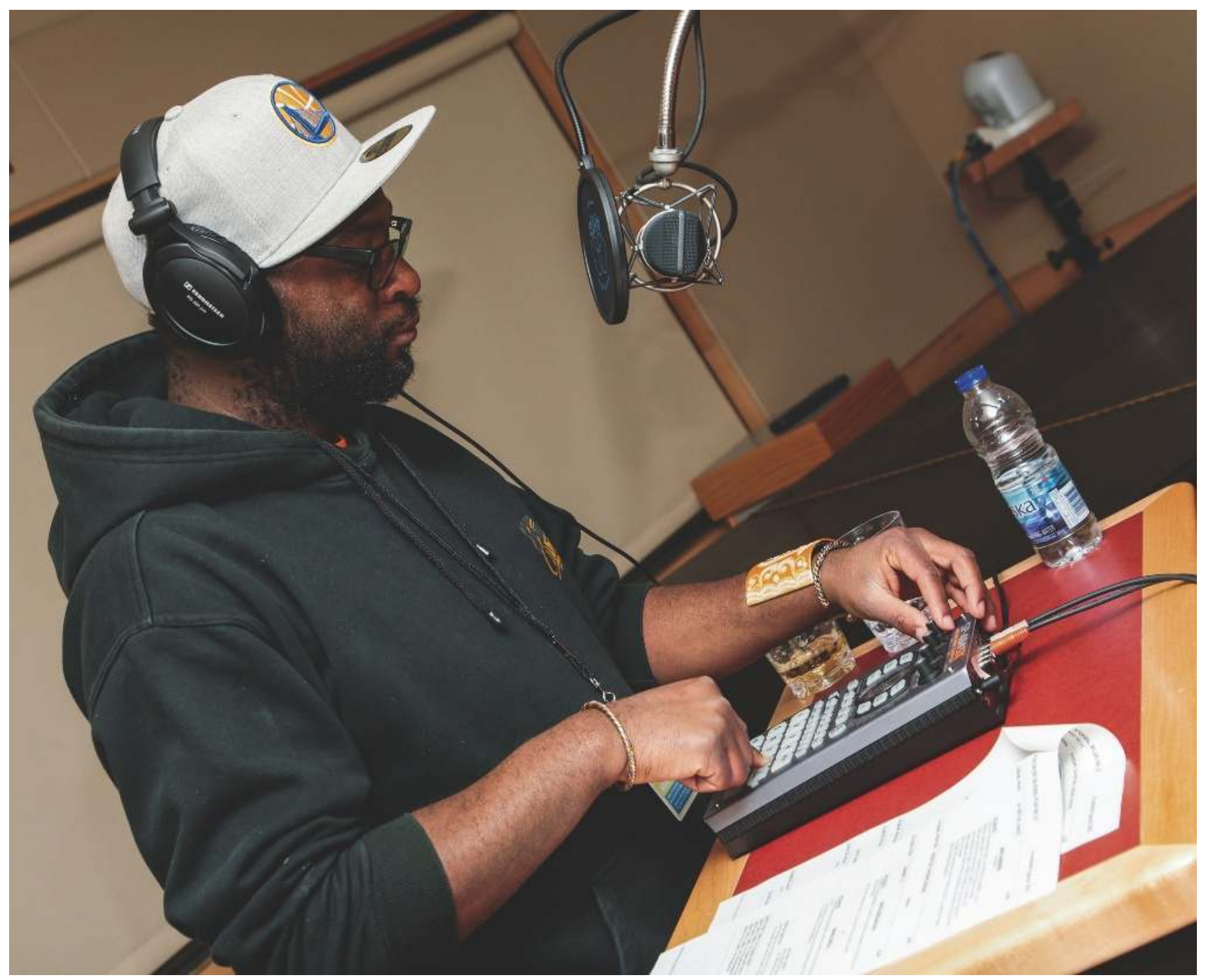

fig. 9

Christian Côté, Engone

Endong, Montréal, 9 janvier

2015 (c) Radio-Canada/

Christian Côté. 
En outre, il apparaît que sans être nécessairement porteurs d'une idéologie patrimoniale ou d'une volonté de faire archive, en collectant, classant et conservant des sonorités préexistantes, ces artistes se font, à plusieurs titres, les créateurs d'archives ordinaires et potentiellement subversives. Plusieurs d'entre eux se positionnent ainsi dans ce champ de force qu'est la production et la transmission d'une vision de l'histoire et d'une mémoire populaire, que ce soit par le recours aux traditions orales et aux instruments rituels, ou par la recréation d'ambiances sonores de l'environnement librevillois. Ils se documentent sur Internet, suivent des conférences en ligne, consultent des ouvrages et entrent parfois en contact avec des intellectuels et des universitaires locaux en vue d'assumer une fonction “d'intellectuel organique» (Gramsci 2001 [1983]). Bien que leur relation au passé ne repose pas sur une recherche méthodique, ils s'emparent de différentes sources historiques et numériques afin de combler un vide laissé par les institutions locales et d'amenuiser l'écart qui sépare les classes populaires des lieux de savoir légitimes.

Finalement, dans le contexte chronophage du Gabon, ce travail de découpage, de sélection et de resignification du sonore amené par le sampling introduit discrètement dans le quotidien des extraits sonores dépositaires de liens avec le passé qui émanent d'une intention de «faire histoire» pour le futur. Les beatmakers contribuent ainsi à peupler l'ordinaire des Librevillois d'une pluralité de sonorités qui font resurgir les voix de minorités bâillonnées ou celles des morts occultées. 
Bibliographie

\section{Appadurai, Arjun}

2003 "Archive and Aspiration", in Brouwer, Joke et Mulder, Arjen (dir.), Information is Alive: Art and Theory on Archiving and Retrieving Data. Rotterdam, V2_Publishing/ NAI Publishers: 14-25.

\section{Artières, Philippe et Kalifa, Dominique}

2002 «Présentation. L'historien et les archives personnelles: Pas à pas ", Sociétés \& Représentations 13: 7-15.

\section{Artières, Philippe et Laé, Jean-François}

2011 Archives personnelles: histoire, anthropologie et sociologie. Paris, Armand Colin.

\section{Aterianus-Owanga, Alice}

2013 "Pratiques musicales, pouvoir et catégories identitaires: anthropologie du rap gaboma. » Thèse de doctorat en anthropologie, université Lumière Lyon II.

2014 « Gaboma, Kainfri, Afropéen. Emboîtements et circulations des catégories identitaires dans le rap gabonais ", Cahiers d'études africaines 216: 945-974.

2016 "Polyphonies du rap", Politique africaine 141.

À paraître "Un "Janus à deux visages". Patrimonialisations des sociétés initiatiques et de discours de la tradition chez quelques musiciens gabonais ", Autrepart 80. Aterianus-Owanga, Alice et Moulard, Sophie (dir.)

\section{Auzanneau, Michelle}

2001 «Le rap, expression de dynamiques urbaines plurilingues. Le cas de Libreville",

Plurilinguismes 18: 11-47.

\section{Béthune, Christian}

2003 Le Rap: une esthétique hors-la-loi. Paris, Autrement.

2011 "Le hip-hop : une expression mineure», Volume! 8 (2) [en ligne], disponible sur: http ://volume. revues.org/2728 (consulté le 3 mai 2016).

\section{Bijsterveld, Karin et Van Dijck, José (dir.)}

2009 Sound Souvenirs: Audio Technologies, Memory and Cultural Practices. Amsterdam, Amsterdam University Press.

\section{Charry, Eric (dir.)}

2012 Hip Hop Africa: New African Music in a Globalizing World. Bloomington, Indiana University Press.

\section{Côté-Lapointe, Simon}

2014 «Archives sonores et création: une pratique à la croisée des chemins ", in Yvon Lemay et Anne Klein, Archives et création: nouvelles perspectives sur l'archivistique. Montréal, université de Montréal, École de bibliothéconomie et des sciences de l'information (EBSI) : 60-84.

\section{Deleuze, Gilles et Guattari, Félix}

1975 Kafka: pour une littérature mineure. Paris, Minuit.

\section{Déon, Maxence}

2011 "L'échantillonnage comme choix esthétique. L'exemple du rap ", Volume! 8 (1) [en ligne], disponible sur: http://volume.revues.org/1307 (consulté le 6 mai 2016).

\section{Derrida, Jacques}

1995 Archive Fever: A Freudian Impression, trad. du français par Eric Prenowitz. Chicago, University of Chicago.

\section{Engandja-Ngoulou, Kanel}

2012 Le Développement des industries culturelles au Gabon. Paris, L'Harmattan.

\section{Fernando, Skiz H.}

2000 The New Beats: culture, musique et attitudes du hip-hop, trad. de l'anglais par Arnaud Réveillon et Jeran-Philippe Henquel. Paris, Éditions de l'Éclat.

\author{
Foucault, Michel \\ 1969 L'Archéologie du savoir. \\ Paris, Gallimard.
}

\section{Foster, Hal}

2004 "An archival impulse", October 110: 3-22.

\section{Gaulier, Armelle}

2015 «La musique du groupe Zebda entre configurations identitaires et pouvoir symbolique", Transposition 5 [en ligne], disponible sur: http :// transposition.revues. org/1398 (consulté le 29 mai 2016).

\section{Gramsci, Antonio}

2001 [1983] Textes, André Tosel (éd.), trad. de l'italien par Jean Bramont et al. Disponible en ligne sur: http://classiques.uqac.ca/ classiques/gramsci_antonio/textes/ textes.html

\section{Guillebaud, Christine et Stoichiţă, Victor A.,}

2013 «Introduction. Constructions sociales de l'humour sonore", Cahiers d'ethnomusicologie 26 [en ligne], disponible sur: http:// ethnomusicologie.revues.org/1970 (consulté le 31 décembre 2015).

\section{Harkins, Paul}

2012 "La transmission perdue et retrouvée», Réseaux 172: 92-118.

\section{Huyssen, Andreas}

2011 La Hantise de l'oubli: essais sur les résurgences du passé, trad. de l'anglais par Julie de Faramond et Justine Malle. Paris, Kimé.

\section{Kosmicki, Guillaume}

2010 «Musique techno, mix, sample», Gradhiva 12: 98-115.

\section{Le Guern, Philippe}

2012 «Irréversible? Musique et technologies en régime numérique ", Réseaux 172: 29-64.

\section{Limat-Letellier, Nathalie et Miguet- Ollagnier, Marie (dir.)}

1998 L'intertextualité.

Besançon, Presses universitaires de Franche-Comté.

\section{Loriot, Charlotte et Roubet, Anne (dir.) \\ 2011 Humoresques 32.}

\section{Martin, Denis-Constant} (dir.)

2010 Quand le rap sort de sa bulle: sociologie politique d'un succès populaire. Paris, Irma/Seteun.

\section{Marshall, Wayne}

2006 « Giving Up Hip-Hop's Firstborn: A Quest for the Real after the Death of Sampling ", Callaloo 29 (3) : 868-892.

\section{Mbembe, Achille}

2002 «The Power of The Archive and Its Limits ", in Carolyn Hamilton et al., Refiguring the Archive. Cape Town, David Philip : 243-282.

\section{Messi Me Nang, Clotaire}

2005 "Les Archives nationales du Gabon. Déliquescence d'une institution documentaire ", Afrique \& histoire 3 : 157-164.

\section{Péan, Pierre,}

2014 Nouvelles Affaires africaines: mensonges et pillages au Gabon. Paris, Fayard.

\section{Potin, Yann}

2015 " Les archives et la matérialité différée du pouvoir. Titres, écrins ou substituts de la souveraineté? ", Pouvoirs 153: 5-21.

\section{Retondah, Guy Steve Touré}

2012 Ezélé! Pierre Akendengué, un cri de liberté. Libreville, Éditions Raponda-Walker. 


\section{Rose, Tricia}

1994 Black Noise: Rap Music and Black Culture in Contemporary America. Hanover/Londres, Wesleyan University Press.

\section{Saucier, Paul Khalil (dir.)}

2011 Native Tongues: an African

Hip-Hop Reader. Trenton, Africa World Press.

\section{Schafer, Raymond Murray}

1979 [1977] Le Paysage

sonore. Toute l'histoire de notre

environnement sonore à travers les

âges, trad. de l'anglais par Sylvette

Gleize. Paris, Jean-Claude Lattès.

\section{Schloss, Joseph Glenn}

2014 Making Beats: the Art

of Sample-Based Hip-Hop.

Middletown, Wesleyan University

Press.

\section{Stoler, Ann Laura}

2010 Along the Archival Grain:

Epistemic Anxieties and Colonial

Common Sense. Princeton,

Princeton University Press.

\section{Swedenburg, Ted}

1997 «Islam in the Mix: Lessons of the Five Percent ", article presenté au colloque d'anthropologie

de l'université de l'Arkansas

(19 février 1997). Disponible en ligne sur: http://comp.uark.

edu/ tsweden/5per.html (consulté

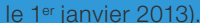

\section{Tindy-Poaty, Juste Joris}

2008 Pierre-Claver Akendengué

ou l'épreuve du miroir. Paris,

l'Harmattan.

\section{Van Dijck, José}

$\mathbf{2 0 0 9}$ «Remembering Songs through Telling Stories: Pop Music as a Resource for Memory", in Karin Bijsterveld et José Van Dijck (dir.), Sound Souvenirs: Audio

Technologies, Memory and Cultural Practices. Amsterdam, Amsterdam University Press: 107-119. page 108 et ci-contre Guy Tillim, Libreville,

2012, diptyque, encre à pigment sur papier coton (c) Guy Tillim. Avec l'aimable autorisation de Stevenson Cape Town et Johannesburg.

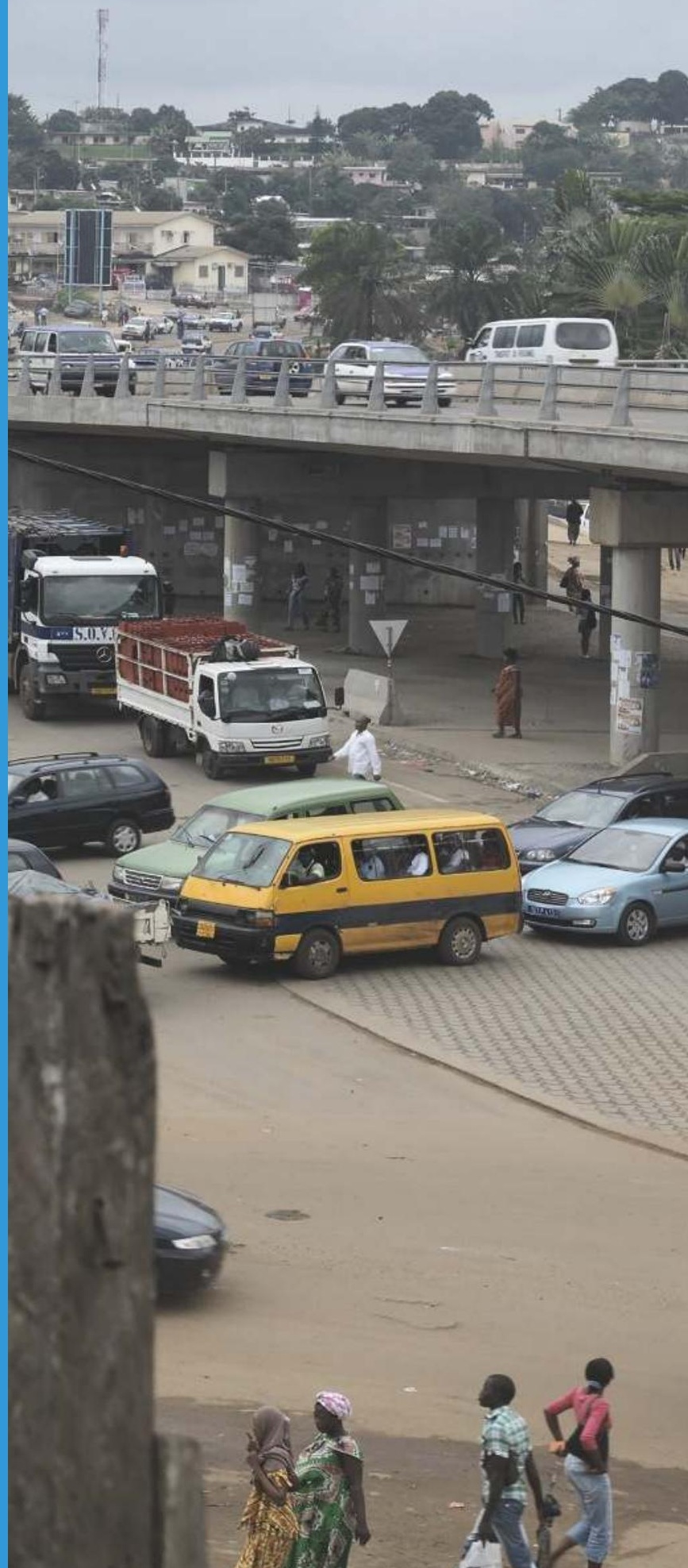

